\title{
Non-modal instability in plane Couette flow of a power-law fluid
}

\author{
R. LIU† AND Q. S. LIU \\ Key Laboratory of Microgravity (National Microgravity Laboratory), Institute of Mechanics, \\ Chinese Academy of Sciences, Beijing, China 100190
}

(Received 18 May 2010; revised 16 January 2011; accepted 19 January 2011;

first published online 26 April 2011)

In this paper, we study the linear stability of a plane Couette flow of a power-law fluid. The influence of shear-thinning effect on the stability is investigated using the classical eigenvalue analysis, the energy method and the non-modal stability theory. For the plane Couette flow, there is no stratification of viscosity. Thus, for the stability problem the stress tensor is anisotropic aligned with the strain rate perturbation. The results of the eigenvalue analysis and the energy method show that the shear-thinning effect is destabilizing. We focus on the effect of non-Newtonian viscosity on the transition from laminar flow towards turbulence in the framework of non-modal stability theory. Response to external excitations and initial conditions has been studied by examining the $\epsilon$-pseudospectrum and the transient energy growth. For both Newtonian and non-Newtonian fluids, it is found that there can be a rather large transient growth even though the linear operator of the Couette flow has no unstable eigenvalue. The results show that shear-thinning significantly increases the amplitude of response to external excitations and initial conditions.

Key words: non-Newtonian flows, transition to turbulence

\section{Introduction}

Shear flows of Newtonian and non-Newtonian fluids are very common in industrial applications as well as in natural processes. In many flow applications, preventing a transition from laminar flow to turbulence is highly desirable. Non-Newtonian viscosity significantly influences the stability of shear flows (Ranganathan \& Govindarajan 2001; Malik \& Hooper 2005; Eldabe, EI-Sabbagh \& EI-Sayed 2007). Thus, changing the non-Newtonian viscosity of fluids in shear flows is an effective way to control the behaviours in the transition from laminar flow to turbulence. In applications, the change of viscosity of a non-Newtonian fluid can be achieved by the addition of 'shear-thinning/shear-thickening' substances, by heating or cooling the walls, or by employing a stratification of viscosity in the direction normal to the wall.

\subsection{Modal and non-modal stability}

Stability and transition to turbulence in shear flows have been studied extensively over the years. Traditionally, a first step in investigating transition is the normal-mode analysis. For this approach, it is assumed that each mode has an exponential time dependence; thus, the base flow is considered to be unstable if an eigenvalue is found in the unstable complex half-plane. In early years, computing the Orr-Sommerfeld 
(OS) equation was a difficult problem; thus, many early works on hydrodynamic problems relied on simplifying assumptions and sophisticated asymptotic techniques (Davey \& Drazin 1969; Davey \& Nguyen 1971). Thomas (1953) used a finite difference scheme to calculate the eigenvalues of the OS equation of plane Poiseuille flow. Orszag (1971) proposed an accurate numerical method, i.e. the Chebyshev spectral method, to solve the same problem as Thomas (1953), which has extended to stability problems for a wide variety of flows including plane Couette flow and pipe Poiseuille flow.

The eigenvalue analysis has successfully predicted the instability behaviours for some fluid systems, such as Rayleigh-Bénard convection and Taylor-Couette flow. However, for other instability problems, particularly the instability in shear flows, this approach fails to match most experimental results. For example, eigenvalue analysis shows that the plane Couette flow and pipe Poiseuille flow are always stable to small disturbances for all Reynolds numbers $(R e)$. However, transition to turbulence is observed for the plane Couette flow at $R e \approx 360$ (Tillmark \& Alfredsson 1992) or 370 (Malerud, Måløy \& Goldburg 1995), and for pipe Poiseuille flow at $R e \approx 2000$ (Wygnanski \& Champagne 1973; Wygnanski, Sokolov \& Friedman 1975). Experiments show that the route to turbulence is highly dependent on the initial conditions and on the continuous forcing that background noise can provide. Such discrepancies between the modal analysis and experiments have been recognized by some authors, and the limiting nature of eigenvalue approach has led to the emergence of a novel way, i.e. the non-modal stability theory.

We will only briefly outline the non-modal theory here and refer the reader to the work by Schmid (2007) and the references therein for further discussion. In the normal-mode analysis, the instability behaviour is inferred from the spectrum of the governing linear operator. However, the spectrum only describes the asymptotic fate of the perturbations and fails to capture short-term characteristics. The main point of departure of the non-modal theory from the traditional eigenvalue analysis is the fact that even if all of the eigenvalues of a linear system are distinct and lie well inside the stable half-plane, inputs to that system may be amplified by substantially larger factors if the linear operator is non-normal. In the non-modal theory, stability is redefined in a broader sense as the response behaviour of the governing equations to general input variables in the form of initial conditions, external forcing and internal uncertainties (Schmid 2007). Trefethen et al. (1993) addressed the general concept of the non-modal stability theory and studied the transient energy growth and the response to external excitations for the plane Poiseuille flow and plane Couette flow. For more mathematics of behaviour of non-normal linear operators, the reader may refer to the book by Trefethen \& Embree (2005). The non-modal theory provided new physical insights into the stability of shear flows; so the transient growth mechanism of non-normal systems has attracted more attentions in the field of hydrodynamic stability. Reddy \& Henningson (1993) have investigated the energy growth of plane Poiseuille and Couette flows in the framework of the non-modal theory. A similar analysis of the transient behaviour of pipe Poiseuille flow has been presented by Schmid \& Henningson (1994). An overview of recent works on the non-modal stability of shear flows can be found in the review article by Schmid (2007).

\subsection{Streaks and bypass transition}

In experiments, streamwise streaks, i.e. narrow regions where the streamwise velocity is larger or smaller than the average, are very commonly observed in plane shear 
flows (Kline et al. 1967; Kiton, Nakabyashi \& Nishimura 2005). These alternating longitudinal high- and low-velocity streaks are independent of the two-dimensional Tollmien-Schlichting (TS) waves. We do not intend here to give a full review of earlier experimental studies. Relevant experimental results on streaky structures in shear flows can be found in the works of Matsubara \& Alfredsson (2001) and Hwang \& Cossu (2010) and the references therein.

For in viscid shear flows, Ellingsen \& Palm (1975) showed that the streamwise velocity component can increase linearly with time for disturbances with no streamwise variation, producing alternating low- and high-velocity streaks in the streamwise velocity component. In laminar shear flows, it is known that streaks have the potential to be largely amplified from streamwise vortices through the 'lift-up' effect (Landahl 1975, 1980). This mechanism works by efficiently extracting momentum from the mean flow and transferring it to the perturbation. Mathematically speaking, the nonmodal theory offers an explanation for why these streamwise streaks are so common: even though these structures are not eigenmodes of the linearized flow problem, they are pseudomodes (Trefethen et al. 1993). Reddy \& Henningson (1993) computed the optimally amplified disturbances in channel flows using a singular value analysis. It is found that initial states with largest transient energy growth in subcritical flows lead to flow structures related to streamwise vortices and streaks that resemble the structures observed in experimental realisations of turbulent flows.

It has been observed that a linearly stable shear flow can exhibit very large energy growth of three-dimensional disturbances. This implies that the nature of the disturbances which appear in shear flows is also incompletely explained by traditional linear stability theory. The reason for the discrepancy between traditional linear stability theory and the experiments has been sought in the framework of nonlinear theories. One example is the secondary instability of TS wave. However, the secondary instability of the TS wave does not seem to completely describe natural transition in plane channel flows (Reddy et al. 1998). These discrepancies between traditional linear and nonlinear theories and experiments have been recognized, and the limiting nature of these approaches has led to intense re-examination of traditional stability theories. An alternative approach is to look directly at the initial value problem, which has attracted the attention of researchers studying transition to turbulence in viscous shear flows. Transition that does not follow the traditional route of exponential instability has been known as 'bypass transition' (Schmid \& Henningson 2001). It is a possible mechanism which appears to reconcile the traditional theory and experimental observations in shear flows. This mechanism is primarily due to linear amplification of disturbances energized by the background mean shear (Butler \& Farrell 1992; Trefethen et al. 1993). It occurs in the absence of nonlinear effect and bypasses the primary/secondary instability.

\subsection{Non-Newtonian effect on stability of shear flow}

The non-Newtonian fluids include polymer solutions, polymer melts, soap solutions, suspensions, emulsions and some biological fluids (Bird, Stewart \& Lightfoot 2002). The physical mechanisms of non-Newtonian fluids are complex; here, we focus on polymeric liquids. Because polymeric liquids contain high-molecular-weight molecules with many internal degrees of freedom, polymer solutions and molten polymers have behaviours qualitatively different from that of Newtonian fluids. One of the most important characteristics of macromolecular fluids is the non-Newtonian viscosity, i.e. the fact that the viscosity $\mu$ changes with the shear rate $\dot{\gamma}$. In many industrial problems, the relation between $\log \mu$ and $\log \dot{\gamma}$ can be described by a well-known constitutive 
equation, i.e. the 'power-law' model $\mu=m \dot{\gamma}^{n-1}$ (Bird, Armstrong \& Hassager 1977). Various forms, such as the Carreau model, of the generalized Newtonian fluid can be found in the book by Bird et al. (1977).

The power-law model has been widely used in engineering and theoretical works. Typical power-law fluids include some colloids, milk, gelatine, blood and liquid cement. Bird et al. (1977) discussed the application of the power-law model in polymeric liquids. When the power-law exponent $n=1$, the fluid is Newtonian. For $n<1$, the fluid is said to be 'pseudoplastic' or 'shear-thinning' and for $n>1$ the fluid is called 'dilatant' or 'shear-thickening'. Shear-thinning fluids are usually solutions of large polymeric molecules in a solvent with smaller molecules, and values of $n$ are in the range of $0.15-0.6$ in general. Most macromolecular fluids are shear-thinning, and shear-thickening fluids are rarely encountered. The parameter $m$ is in units of $\mathrm{Pa}^{n}$. Typical parameters for aqueous solutions are given in Bird et al. (2002). At the temperature changing from 293 to $333 \mathrm{~K}$, the magnitude of $m$ is in the range of $10^{-1}-10^{2}$.

Although the generalized Newtonian fluid is of great value in engineering problems, its use is strictly limited to steady-state shearing flows. For unsteady non-Newtonian flows, the elastic response of the fluid becomes important. The Maxwell model or the Jeffreys model is widely used to describe linear viscoelastic fluids (Bird et al. 1977). The Oldroyd models can be used to describe nonlinear viscoelastic fluids (Bird et al. 1977).

Non-Newtonian effect of different types of fluids on the stability of complex shear flows has been extensively studied for recent decades. Ranganathan \& Govindarajan (2001) have investigated the stability of channel flow by locating the viscosity-stratified fluid layer. Roberts \& Kumar (2006) have studied the stability of creeping Couette flow of a power-law fluid past a deformable solid. Nouar \& Frigaard (2009) have studied the stability of plane Couette-Poiseuille flow of a shear-thinning fluid using the Carreau-Yasuda model. Pinarbasi \& Imal (2005) have investigated the effect of viscous heating on the linear stability of Poiseuille flow of a Carreau fluid with a temperature-dependent viscosity described by an Arrhenius law. Eldabe et al. (2007) examined the effect of shear-thinning and shear-thickening on the stability of the plane Couette flow with viscous heating for a power-law fluid that obeys the Arrhenius law. However, these works just mentioned are in the framework of traditional normalmode analysis. Thus, the results in these works only provide us the asymptotic behaviours of eigenmodes.

In order to control non-Newtonian flows, it is crucial to know how non-Newtonian effect influences the transient behaviour of disturbances from laminar flow to turbulence. Recently, some authors have extended the scope of non-modal stability for non-Newtonian fluids. Bergström (2003) has investigated the transient growth of small disturbances in a pipe flow of a Jeffrey fluid. Nouar et al. (2007) have considered the modal and non-modal stabilities of plane Bingham-Poiseuille flow of a viscoplastic fluid. Hoda, Jovanović \& Kumar (2008, 2009) have examined the frequency response in streamwise-constant channel flows of Oldroyd-B fluids from an input-output of view. The authors have analysed in detail the ensemble-average energy density and the componentwise frequency response to spatiotemporal body forces. These two studies by Hoda et al. (2008, 2009) have extended the previous studies (Jovanovic 2004; Jovanovic \& Bamieh 2005), where similar analyses have been done for Newtonian fluids. Works just mentioned presented the effect of viscoelasticity or viscoplasticity on the non-modal stability. However, a careful look at previous works shows that studies on effect of non-Newtonian viscosity on the non-modal transient behaviour are very 


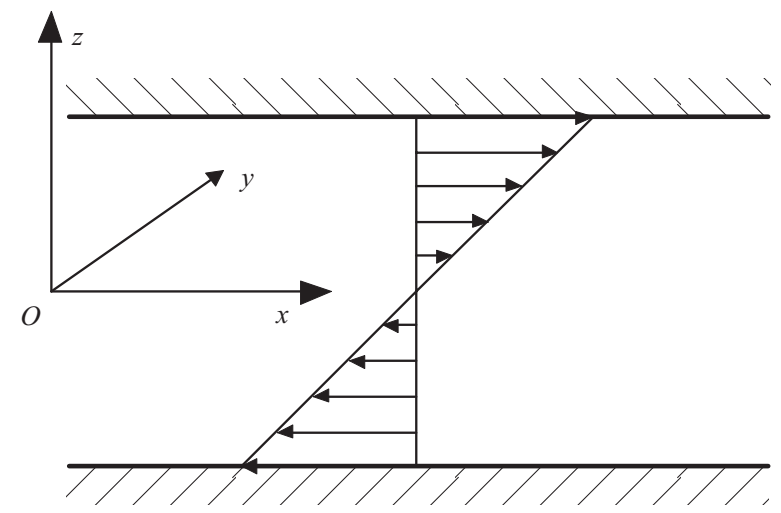

Figure 1. Geometry and coordinate system for a plane Couette flow of a power-law fluid.

limited. To our knowledge, two recent papers (Chikkadi, Sameen \& Govindarajan 2005; Nouar, Bottaro \& Brancher 2007) have examined the influence of shear-thinning effect on the transient growth of a plane Poisueille flow. Chikkadi et al. (2005) have studied two typical cases of channel flow. One is the plane Poiseuille flow of a Carreau fluid, while the other is two miscible fluids of equal densities but different viscosities. Rather unexpectedly, the authors found that a stratification of viscosity alone does not affect transient growth; moreover, the transient growth is only slightly influenced by the shear-thinning effect. The result seems quite important to the requirement of preventing transition to turbulent. However, we should note that the result is only valid for neglecting the viscosity perturbations. Nouar et al. (2007) have revisited the stability of shear-thinning fluids. The authors have accounted for a non-vanishing viscosity disturbance and obtained significantly different results to that reported by Chikkadi et al. (2005). We defer further discussions on the physics of the viscosity disturbance to a later section in which the mathematical description is established. Motivated by recent works mentioned above (Chikkadi et al. 2005; Nouar et al. 2007), an open question should be asked, does shear-thinning effect significantly influence the transient behaviour for other type of shear flows.

In the present work, we aim to study the non-modal instability in a plane Couette flow of a power-law fluid. The purpose is to provide physical insights into the effect of shear-thinning on the non-modal behaviours, including response to excitations and transient energy growth of initial conditions. This type of flow allows us to isolate the influence of the anisotropy of the stress tensor associated with the perturbation. In other situations, like in the plane Poiseuille flow, three effects arise from the rheology of the fluid: (i) modification of the velocity gradient, (ii) viscosity stratification and (iii) anisotropy of the stress tensor. In a global way, it is not easy to separate the different influences.

The paper is organized as follows. In $\S 2$, mathematical formulation of the physical model is presented. The numerical method for solving the eigenvalue problem is presented in $\S 3$. In $\S \S 4$ and 5, we present the results and discussions. Finally, in $\S 6$, we summarize the results and present the conclusions.

\section{Mathematical formulation}

Figure 1 illustrates the physical model of the present study. Consider a flow of a power-law fluid bounded by two infinite parallel plates moving in opposite directions 
with velocities $\pm U$ at $z= \pm d$. The parallel base flow $\overline{\boldsymbol{u}}(z)=[\bar{u}(z), 0,0]$ only depends on $z$. The equations governing the evolution of fluid flow are the Navier-Stokes equations. For an incompressible non-Newtonian fluid, the equations are expressed as

$$
\begin{gathered}
\nabla \cdot \boldsymbol{u}=0 \\
\frac{\partial \boldsymbol{u}}{\partial t}+\boldsymbol{u} \cdot \nabla \boldsymbol{u}=-\frac{1}{\rho} \nabla p+\frac{1}{\rho} \nabla \cdot \boldsymbol{\tau}
\end{gathered}
$$

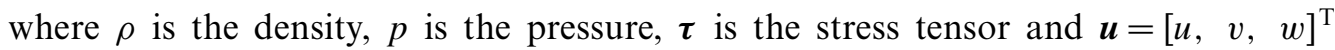
is the velocity vector. $u, v$ and $w$ are the velocity components in the streamwise $x$, spanwise $y$ and normal to wall $z$ directions, respectively.

For incompressible non-Newtonian fluids, the expression for the stress tensor is given by the generalized Newtonian model:

$$
\boldsymbol{\tau}=\mu \dot{\boldsymbol{\gamma}}, \quad \text { with } \mu=\mu(\dot{\gamma}) .
$$

Here, the symbol $\dot{\gamma}=\nabla \boldsymbol{u}+(\nabla \boldsymbol{u})^{\mathrm{T}}$ is the rate of strain tensor and $\mu(\dot{\gamma})$ is the nonNewtonian viscosity. The magnitude of the rate of strain tensor is

$$
\dot{\gamma}=\sqrt{\frac{1}{2}(\dot{\gamma}: \dot{\gamma})} .
$$

In the present study, we choose the two-parameter power-law expression for $\mu(\dot{\gamma})$,

$$
\mu=m \dot{\gamma}^{n-1},
$$

in which $m$ and $n$ are the constants characterizing the fluid.

The evolution equations for the disturbances can be derived by considering a basic state $(\overline{\boldsymbol{u}}, \bar{p})$ and the perturbed state $(\boldsymbol{u}, p)=\left(\overline{\boldsymbol{u}}+\boldsymbol{u}^{\prime}, \bar{p}+p^{\prime}\right)$. For both states, the Navier-Stokes equations are satisfied.

\subsection{Basic state}

In the basic state, the velocity and the magnitude of $\dot{\gamma}$ are

$$
\overline{\boldsymbol{u}}=\left(\frac{U z}{d}, 0,0\right), \quad \overline{\dot{\gamma}}=\frac{U}{d} .
$$

The rates of strain tensor and stress tensor, respectively, are

$$
\overline{\dot{\gamma}}=\left(\begin{array}{lll}
0 & 0 & \frac{U}{d} \\
0 & 0 & 0 \\
\frac{U}{d} & 0 & 0
\end{array}\right) \quad \text { and } \quad \overline{\boldsymbol{\tau}}=\left(\begin{array}{lll}
0 & 0 & m\left(\frac{U}{d}\right)^{n} \\
0 & 0 & 0 \\
m\left(\frac{U}{d}\right)^{n} & 0 & 0
\end{array}\right) .
$$

For a plane Poiseuille flow, both the distributions of the basic velocity and the stress tensor are the functions of $z$ and are dependent on the index $n$. For a plane Couette flow, the power-law fluid has the same basic velocity with the Newtonian fluid, i.e. the velocity of the basic state is a linear function of $z$ and is independent of the index $n$. Moreover, the rate of strain tensor and the stress tensor of basic state are constants. 


\subsection{Linearized equations}

Subtracting the equations for the basic and perturbed state, the linearized evolution equations for disturbances can be written as

$$
\begin{aligned}
\nabla \cdot \boldsymbol{u}^{\prime} & =0 \\
\frac{\partial \boldsymbol{u}^{\prime}}{\partial t}+\overline{\boldsymbol{u}} \cdot \nabla \boldsymbol{u}^{\prime}+\boldsymbol{u}^{\prime} \cdot \nabla \overline{\boldsymbol{u}} & =-\frac{1}{\rho} \nabla p^{\prime}+\frac{1}{\rho} \nabla \cdot \boldsymbol{\tau}^{\prime}
\end{aligned}
$$

where $\boldsymbol{\tau}^{\prime}$ is the shear stress perturbation given by $\boldsymbol{\tau}^{\prime}=\bar{\mu} \dot{\boldsymbol{\gamma}}^{\prime}+\mu^{\prime} \overline{\boldsymbol{\gamma}}$. In (2.9), $\nabla \cdot \boldsymbol{\tau}^{\prime}$ can be expressed as

$$
\nabla \cdot \boldsymbol{\tau}^{\prime}=\nabla \cdot\left(\bar{\mu} \dot{\gamma}^{\prime}\right)+\nabla \cdot\left(\mu^{\prime} \overline{\dot{\gamma}}\right) .
$$

The first term of the right-hand side of (2.10) is an analogy of the Newtonian fluid. Since there is no stratification of viscosity, it can be shown that

$$
\nabla \cdot\left(\bar{\mu} \cdot \boldsymbol{\gamma}^{\prime}\right)=\bar{\mu} \nabla^{2} \boldsymbol{u}^{\prime} .
$$

The second term of the right-hand side of (2.10) denotes the departure from Newtonian behaviour. It can also be shown that

$$
\nabla \cdot\left(\mu^{\prime} \overline{\boldsymbol{\gamma}}\right)=(n-1) \bar{\mu}\left(\frac{\partial \gamma_{x z}^{\prime}}{\partial z} \boldsymbol{e}_{x}+\frac{\partial \gamma_{x z}^{\prime}}{\partial x} \boldsymbol{e}_{z}\right),
$$

in which $\gamma_{x z}^{\prime}=\partial w^{\prime} / \partial x+\partial u^{\prime} / \partial z$. One can notice that this additional term corresponds to an anisotropic shear stress perturbation. For a general shear flow, like the plane Poiseuille flow, the rate of strain tensor varies in the $z$-direction. Consequences of shear-thinning are the modification of the basic velocity, the stratification of viscosity and the anisotropy of stress perturbation. In general, it is difficult to separate these effects. In order to isolate some of these effects, the disturbance of viscosity could only be artificially neglected (Nouar et al. 2007). For the plane Couette flow, there is no stratification of viscosity, and the basic velocity is independent of $n$. This allows us to isolate the influence of the anisotropy of the stress tensor associated with the perturbation.

\subsection{The Velocity-vorticity formulation}

The controlling equations are non-dimensionalized with the length scale $d$, the velocity scale $U$ and the time scale $d / U$. We introduce the vorticity

$$
\eta=\frac{\partial v}{\partial x}-\frac{\partial u}{\partial y} \text {. }
$$

Using the continuity equation and eliminating the pressure, we can arrive at the OS and Squire equations. We introduce the Fourier mode

$$
[w(x, y, z, t), \eta(x, y, z, t)]=[w(z, t), \eta(z, t)] \exp (\mathrm{i} \alpha x+\mathrm{i} \beta y),
$$

in which $\alpha$ and $\beta$ are the streamwise and the spanwise wavenumbers, respectively. The governing equations are expressed as

$$
\begin{aligned}
& \left(\frac{\partial}{\partial t}+\mathrm{i} \alpha \bar{u}\right)\left(D^{2}-k^{2}\right) w-\mathrm{i} \alpha \frac{\mathrm{d}^{2} \bar{u}}{\mathrm{~d} z^{2}} w=\frac{1}{R e}\left(D^{2}-k^{2}\right)^{2} w+\frac{n-1}{\operatorname{Re}} \frac{\alpha^{2}}{k^{2}}\left(D^{2}+k^{2}\right)^{2} w \\
& +\frac{n-1}{\operatorname{Re}} \frac{\alpha \beta}{k^{2}}\left(D^{2}+k^{2}\right) D \eta
\end{aligned}
$$




$$
\left(\frac{\partial}{\partial t}+\mathrm{i} \alpha \bar{u}\right) \eta=\mathrm{i} \beta \frac{\mathrm{d} \bar{u}}{\mathrm{~d} z} w+\frac{1}{R e}\left(D^{2}-k^{2}\right) \eta+\frac{n-1}{\operatorname{Re}} \frac{\alpha \beta}{k^{2}}\left(D^{2}+k^{2}\right) D w+\frac{n-1}{\operatorname{Re}} \frac{\beta^{2}}{k^{2}} D^{2} \eta .
$$

At $z= \pm 1$, the boundary conditions are

$$
w=D w=\eta=0,
$$

where $D=\partial / \partial z$ and $k^{2}=\alpha^{2}+\beta^{2}$. The Reynolds number Re is defined as

$$
R e=\frac{\rho U d}{m}\left(\frac{d}{U}\right)^{n-1}
$$

The OS equation and the Squire equation can be expressed in the vector form:

$$
\mathscr{B} \frac{\partial}{\partial t} \Psi=\mathscr{A} \Psi,
$$

in which $\Psi=(w, \eta)^{\mathrm{T}}$ and the matrices $\mathscr{A}$ and $\mathscr{B}$ are

$$
\mathscr{B}=\left[\begin{array}{ll}
D^{2}-k^{2} & 0 \\
0 & 1
\end{array}\right], \quad \mathscr{A}=\left[\begin{array}{ll}
\mathscr{L}_{O S} & \mathscr{L}_{d} \\
\mathscr{L}_{c} & \mathscr{L}_{S q}
\end{array}\right] .
$$

The operators $\mathscr{L}_{O S}, \mathscr{L}_{S q}, \mathscr{L}_{c}$ and $\mathscr{L}_{d}$ can be written as

$$
\begin{gathered}
\mathscr{L}_{O S}=-\mathrm{i} \alpha \bar{u}\left(D^{2}-k^{2}\right)+\mathrm{i} \alpha \frac{\mathrm{d}^{2} \bar{u}}{\mathrm{~d} z^{2}}+\frac{1}{R e}\left(D^{2}-k^{2}\right)^{2}+\frac{n-1}{R e} \frac{\alpha^{2}}{k^{2}}\left(D^{2}+k^{2}\right)^{2} \\
\mathscr{L}_{S q}=-\mathrm{i} \alpha \bar{u}+\frac{1}{R e}\left(D^{2}-k^{2}\right)+\frac{n-1}{\operatorname{Re}} \frac{\beta^{2}}{k^{2}} D^{2} \\
\mathscr{L}_{c}=\mathrm{i} \beta \frac{\mathrm{d} \bar{u}}{\mathrm{~d} z}+\frac{n-1}{\operatorname{Re}} \frac{\alpha \beta}{k^{2}}\left(D^{2}+k^{2}\right) D \\
\mathscr{L}_{d}=\frac{n-1}{\operatorname{Re}} \frac{\alpha \beta}{k^{2}}\left(D^{2}+k^{2}\right) D .
\end{gathered}
$$

At last, we obtain the linear initial value problem

$$
\frac{\partial}{\partial t} \Psi=-i \mathscr{L} \Psi
$$

in which $\mathscr{L}=\mathrm{i} \mathscr{B}^{-1} \mathscr{A}$.

\section{Numerical method}

In the present paper, we will examine the linear stability, the response to external excitations and initial conditions. We need to compute the eigenvalues and eigenvectors of the operator $\mathscr{L}$, the pseudospectrum and the numerical range, and the energy growth function $G(t)$.

We begin with the computation of eigenvalue and eigenvectors of $\mathscr{L}$. A Chebyshev collocation method is implemented to solve the eigenvalue problem. The variables $w$ and $\eta$ are expanded as

$$
w=\sum_{n=0}^{N} \hat{w}_{n} T_{n}(z), \quad \eta=\sum_{n=0}^{N} \hat{\eta}_{n} T_{n}(z),
$$


in which $T_{n}$ denotes the $n$th Chebyshev polynomial. Using the Chebyshev series (3.1), the governing equations can be written in the vector form

$$
\frac{\mathrm{d}}{\mathrm{d} t} \hat{\Psi}=-\mathrm{i} \hat{\mathscr{L}} \hat{\Psi}
$$

in which $\hat{\Psi}=\left[\hat{w}_{0}, \ldots, \hat{w}_{N}, \hat{\eta}_{0}, \ldots, \hat{\eta}_{N}\right]^{\mathrm{T}}$ and $\hat{\mathscr{L}}$ arises from the discretization of $\mathscr{L}$. This system of equations is required to solve for $2 N+2$ unknowns. The numerical method for the general eigenvalue problem in the form $\boldsymbol{A} \boldsymbol{x}=\omega \boldsymbol{B} \boldsymbol{x}$ has been described by Canuto et al. (1993) and Schmid \& Henningson (2001). The OS equation is of fourth order; so the equation is applied at $N-3$ internal collocation points and at each boundary the equations are replaced by two boundary conditions. The Squire equation is of second order, and it is applied at $N-1$ internal collocation points with only one boundary condition applied at each boundary. The details of imposing boundary conditions in the collocation method are described by Canuto et al. (1993). When computing the spectrum, $N=80 \sim 160$ is enough to obtain satisfactory results. By varying the number of polynomials, $N$, it is found that 80 polynomials usually yield eight decimal places of accuracy, for the $R e$ values we are working with. For example, at $\alpha=1, R e=1000$ with $n=1.0$, the leading eigenvalue $\omega$ for $N=80,90$ and 100 is always $0.79751396-0.11790537 i$. In our computation, for low Reynolds number and large value of $n$, we choose $N=80$. For high Reynolds number and small value of $n$, because the vicinity of the intersection of the spectrum becomes highly sensitive to perturbations, we need higher order expansions to compute the problem. After we solve the eigenvalue problem, an eigenfunction expansion method can be used to compute the transient energy growth and the response to external excitations. For the details of the computational procedure for non-modal stability, the reader may refer to the works by Reddy \& Henningson (1993) and Schmid \& Henningson (2001).

\section{Asymptotic behaviour, condition for no energy growth}

For the plane Couette flow, we are interested in the asymptotic long time behaviour and the condition of no energy growth of the disturbances. When considering the long time behaviour, the disturbances can be assumed to vary exponentially with time, i.e. $[w(z, t), \eta(z, t)]=[\tilde{w}(z), \tilde{\eta}(z)] \exp (-\mathrm{i} \omega t)$, in which $\omega$ is the complex frequency, and the imaginary part of $\omega$, denoted by $\omega_{i}$, is the temporal growth rate. For a linear evolution equation $\mathrm{d} \Psi / \mathrm{d} t=-\mathrm{i} \mathscr{L} \Psi$, the long time behaviour can be described by the eigenvalues of the linear operator $\mathscr{L}$. The linear stability analysis has shown that the plane Couette flow of a Newtonian fluid is linearly stable for all Reynolds numbers (Romanov 1973).

We begin with examining the effect of the power-law index, $n$, on the long time behaviour of the flow. The growth rates of the least stable mode are presented for spanwise-independent $(\beta=0)$ and streamwise-independent $(\alpha=0)$ disturbances using a representative range of parameters. In figures $2(a)$ and $2(b)$, the curves of growth rate, $\omega_{i}$, versus the streamwise wavenumber $\alpha$ with various $n$, are plotted at $R e=1000$ and $R e=3000$ for spanwise-independent disturbances. For each curve in these two figures, the largest growth rate occurs at the longwave limit of $\alpha \rightarrow 0$, and the growth rate decreases with the increase of $\alpha$. In the longwave range, the curves with various $n$ are almost undistinguishable. At larger wavenumber $\alpha$ for $n \leqslant 1$, it is apparent that more shear-thinning fluids are less stable. As shown in figure $2(a)$ for a lower Reynolds number of $R e=1000$, the curves of $n=1.0$ and 1.5 are almost undistinguishable at 

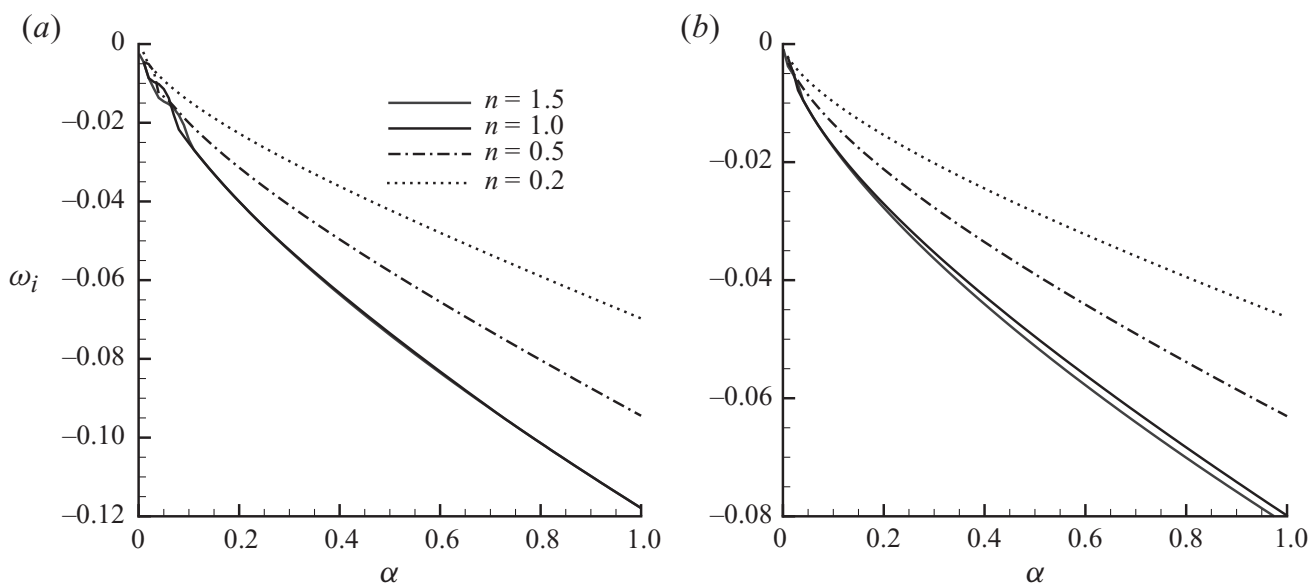

FIGURE 2. Temporal growth rates of spanwise-independent disturbances $(\beta=0)$ versus the streamwise wavenumber $\alpha$ at $(a) \operatorname{Re}=1000 ;(b) \operatorname{Re}=3000$.
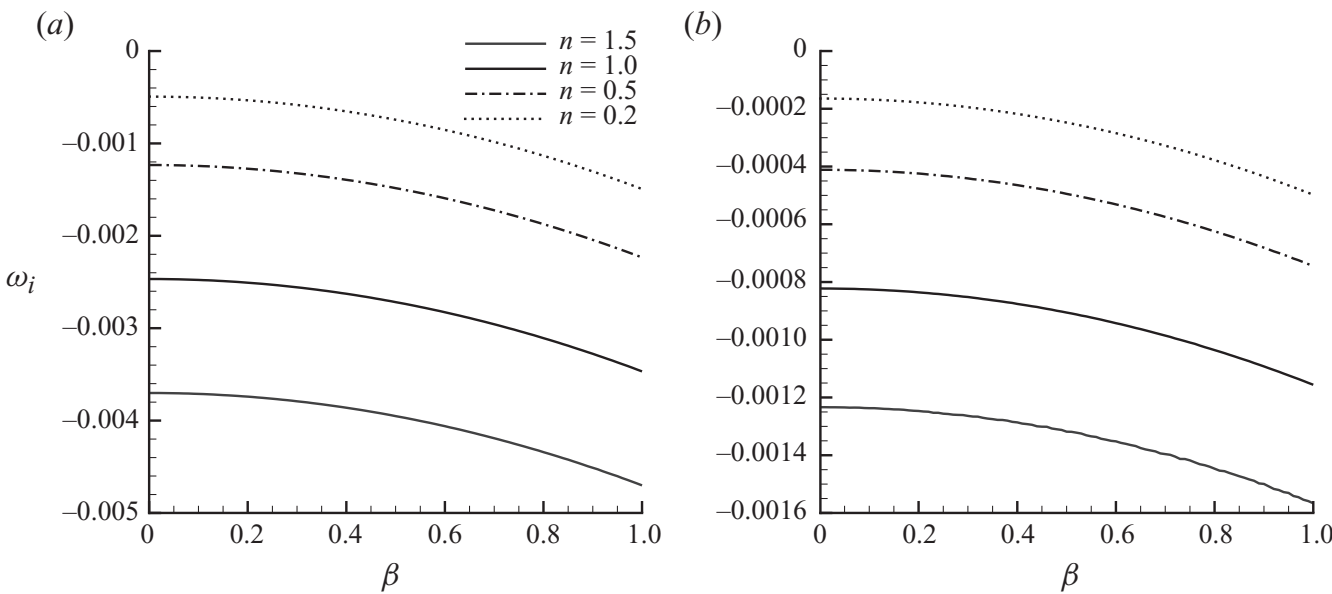

FIGURE 3. Temporal growth rates of streamwise-independent disturbances $(\alpha=0)$ versus the spanwise wavenumber $\beta$ at (a) $R e=1000 ;(b) R e=3000$.

most of wavenumbers. In figure $2(b)$ for a higher Reynolds number of $R e=3000$, the curve of $n=1.5$ is slightly more stable than that of $n=1.0$. It seems that, in the medium or shortwave range, the temporal growth rates of the spanwise-independent disturbances are significantly influenced by shear-thinning effect; however, they are insensitive to shear-thickening effect in the longwave range.

The first rigorous proof of the linear stability of the plane Couette flow was given by Romanov (1973). The author showed that all modes are always damped for all values of the Reynolds number. In figure 2 , the growth rates at all wavenumbers are negative for power-law fluids. We have computed the eigenvalues at higher Reynolds numbers and found no unstable mode. This suggests that, like the Newtonian case, the plane Couette flow of a power-law fluid is always stable for all Reynolds numbers.

In figures $3(a)$ and $3(b)$, the curves of growth rate $\omega_{i}$ of streamwise-independent disturbances versus the spanwise wavenumber $\beta$ are plotted at $R e=1000$ and $R e=3000$. In these two figures, the temporal growth rate of each curve decreases 

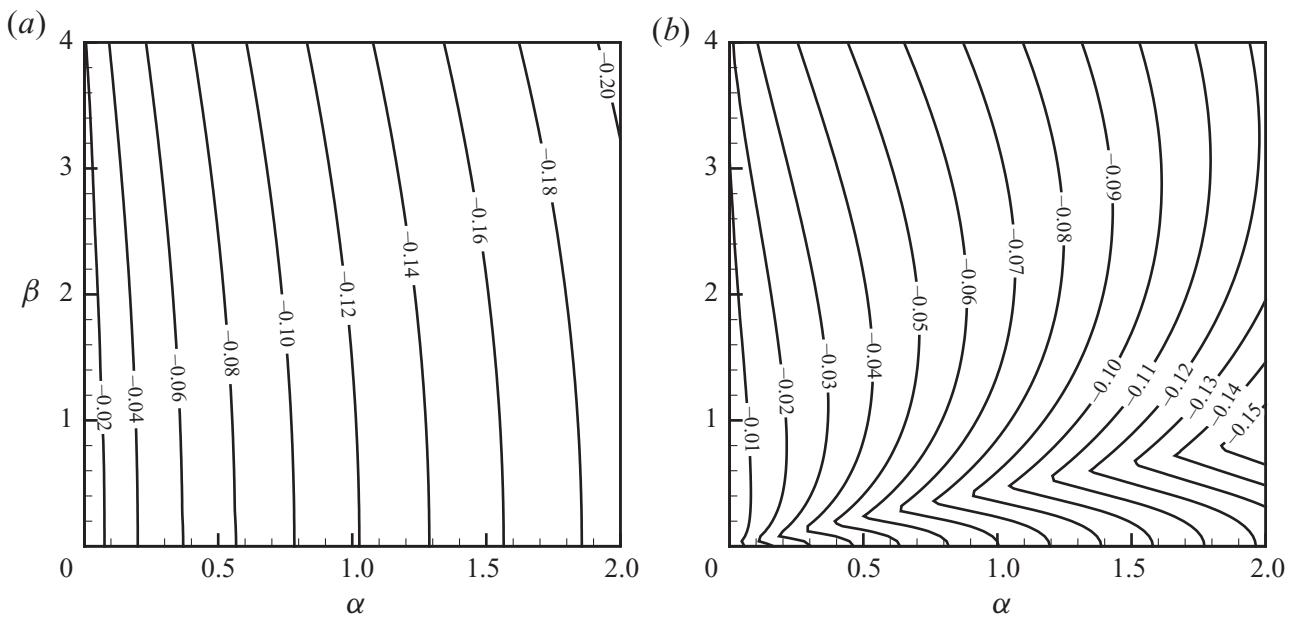

FIGURE 4. Temporal growth rates of oblique disturbances in the $\alpha-\beta$ plane at $R e=1000$ for $(a) n=1 ;(b) n=0.2$.

with the increase of the spanwise wavenumber $\beta$. With the increase of $n$, the temporal growth rate significantly decreases at all wavenumbers. This result shows that the shear-thinning/shear-thickening effect is destabilizing/stabilizing for the streamwiseindependent disturbances. This result is significantly different from that for the spanwise-independent disturbances in figure 2 , in which the shear-thickening effect only slightly influences the temporal growth rate.

In order to know the effect of the index $n$ on the temporal growth rate of oblique disturbances, we plot in figure 4 the level lines of the growth rate in the $\alpha-\beta$ plane for presentative parameters. As shown in figure 4, all the temporal growth rates are negative in the $\alpha-\beta$ plane. This result indicates that all three-dimensional disturbances are linearly stable at $R e=1000$. As shown in figure 4(a) for $n=1$, the temporal growth rate decreases with the increase of $\alpha$ or $\beta$ at each point in the $\alpha-\beta$ plane. For the shear-thinning fluid of $n=0.2$, as shown in figure $4(b)$, the structure of the level lines is significantly different from that in figure $4(a)$ for $n=1$. It is obvious that each level line is contorted. At the kink points, when fixing $\alpha$, the temporal growth rate reaches the local minimum.

To interpret the effect of $n$ on the stability, it is helpful to examine the energy budget of the OS equation. The OS equation is multiplied by $w^{*}$ and integrated in $z$ from -1 to 1 ; it is obtained that

$$
\begin{aligned}
& \omega_{i}\left\langle|D w|^{2}+\alpha^{2}|w|^{2}\right\rangle=\alpha\left\langle D \bar{u}\left(w_{r} D w_{i}-w_{i} D w_{r}\right)\right\rangle-\frac{1}{R e}\left\langle\bar{\mu}\left(4 \alpha^{2}|D w|^{2}+\left|D^{2} w+\alpha^{2} w\right|\right)\right\rangle \\
& +\frac{1}{R e}\left\langle\left(\bar{\mu}-\mu_{t}\right)\left|D^{2} w+\alpha^{2} w\right|^{2}\right\rangle,
\end{aligned}
$$

where $\langle\cdot\rangle=\int_{-1}^{1}(.) \mathrm{d} z$, and

$$
\mu_{t}=\mu(\bar{u})+\frac{\mathrm{d} \mu}{\mathrm{d} \dot{\gamma}_{z x}}(\bar{u}) \dot{\gamma}_{z x}(\bar{u})
$$

is termed the tangent viscosity. For a power-law fluid, $\bar{\mu}-\mu_{t}=(1-n) \overline{\dot{\gamma}}^{n-1}$. The second term on the right-hand side of (4.1) which arises from viscosity stratification is negative and stabilizing. The third term on the right-hand side arises from viscosity 
perturbation. For shear-thinning fluids, this term is positive-definite. It reduces the viscous dissipation, compared to the case with no viscosity perturbations, and plays a destabilizing role. For a plane Couette flow, with the increase of shear-thinning, the value of the third term increases. Nouar et al. (2007) showed that increasing shearthinning effect stabilizes the Poiseuille flow in the case of the time constant $\lambda$ being large. However, for the Couette flow the present results show that the shear-thinning effect is destabilizing. Nouar et al. (2007) stated that the main factor determining stability or instability of the flow is the exchange of energy between base flow and perturbation, which is driven by the phase change between the two fluctuating velocity components, caused by the viscosity stratification. When the viscosity fluctuation is artificially forced to zero, a fictitious stabilization is observed. For the Poiseuille flow, the variation of the index $n$ will result in both viscosity stratification and viscosity fluctuation. For the Poiseuille flow, the increase of shear-thinning has a dual effect on the stability, i.e. the viscosity stratification is stabilizing and the viscosity fluctuation is destabilizing. Nevertheless, the effect of viscosity stratification is dominant; so the total effect of shear-thinning is stabilizing. For the Couette flow, the increase of shearthinning significantly increases the viscosity perturbation; however, it has no effect on the viscosity stratification. So, for the Couette flow, the shear-thinning has only destabilizing effect. For shear-thickening fluids, the third term on the right-hand side of (4.1) is negative-definite. With the increase of shear-thickening, the system becomes more stable.

In this subsection, we are also interested in the condition for which there is no growth of the perturbation kinetic energy. Energy methods give conditions for no energy growth. Using general properties of the inner product, we have

$$
\frac{\mathrm{d}\|\Psi\|^{2}}{\mathrm{~d} t}=2 \operatorname{Im}(\mathscr{L} \Psi, \Psi) .
$$

There is no energy growth if and only if the eigenvalues of $\left(\mathscr{L}-\mathscr{L}^{*}\right) / 2$ lie in the lower half-plane (Reddy \& Henningson 1993). The energy method shows that there is no energy growth if the Reynolds number is less than $R e_{g}$. In order to determine the critical energy Reynolds number $R e_{g}$, two methods can be chosen. One way is to compute the eigenvalues of $\left(\mathscr{L}-\mathscr{L}^{*}\right) / 2$, and the other is to solve the optimization problem using a variational method. The details of the variational method can be found in the works by Reddy \& Henningson (1993), Schmid \& Henningson (2001) and Nouar et al. (2007).

We will examine the effect of $n$ on the critical energy Reynolds number $R e_{g}$, below which the disturbance kinetic energy decays monotonically in time. Figures $5(a)$ and $5(b)$ present the critical energy Reynolds number versus the wavenumber for spanwiseand streamwise-independent disturbances with various $n$. For the Newtonian case of $n=1$, the critical value of Reynolds number for streamwise-independent disturbances is $R e \approx 21$ at the wavenumber of $\beta \approx 1.5$. This result is quantitatively the same as that of (Schmid \& Henningson 2001, see figure 5.11). As shown in figures 5(a) and 5(b), the influence of $n$ on the critical value of the Reynolds number is qualitatively similar for these two cases. With the increase of $n$, the plane Couette flow becomes more stable and the corresponding critical wavenumber decreases. Comparing the curves with the same value of $n$ in figures $5(a)$ and $5(b)$, it is shown that the spanwise-independent disturbances are more stable than the streamwise-independent ones.

The energy Reynolds numbers are far below the Reynolds numbers at which transition to turbulence is observed in experiments. This is mainly due to the nonnormal nature of the linearized Navier-Stokes equations for shear flows. In order to 

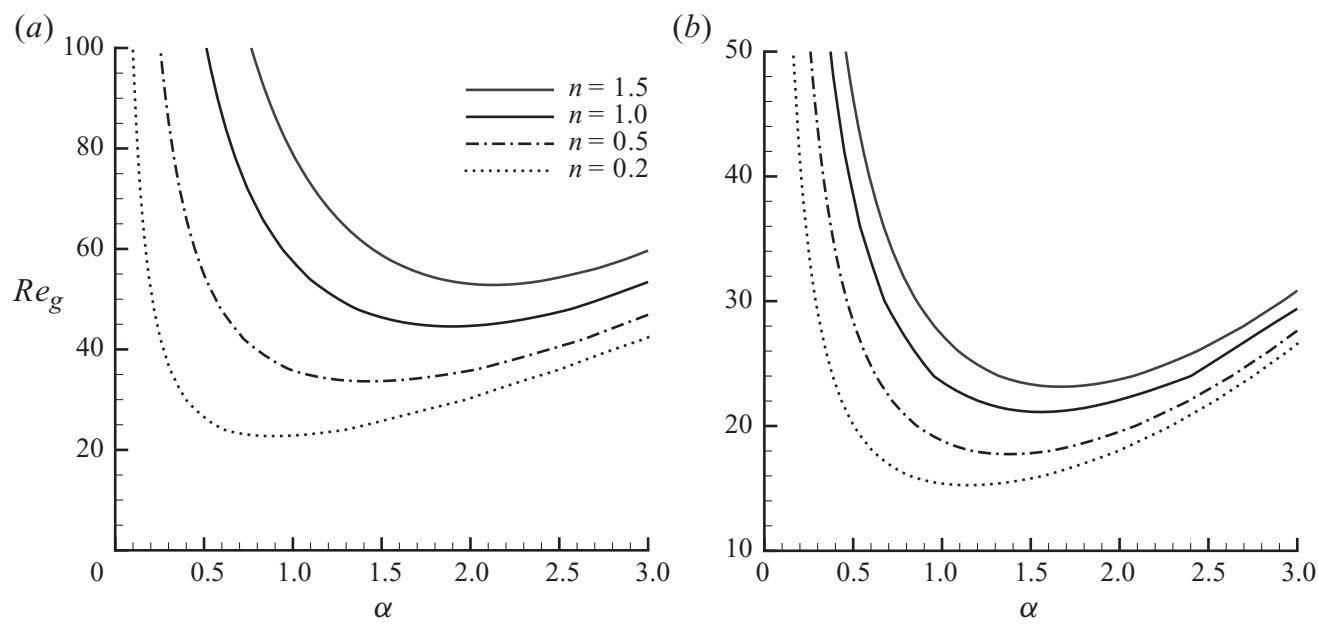

FIGURE 5. Critical energy Reynolds number versus the wavenumber for (a) spanwise-independent disturbances; $(b)$ streamwise-independent disturbances.

know the transient behaviour of the plane Couette flow, we will study the non-normal stability of the present problem.

\section{Transient behaviour and non-modal stability}

In the non-modal theory, two types of problems are of particular interest, i.e. the response to external excitations and the transient energy growth of initial conditions. For the former, the norm of resolvent denotes the maximum amplification of external excitations; for the latter, the growth function $G(t)$ identifies the optimal growth of energy at time $t$. Before we present the results, it is helpful to discuss the mechanisms of the influence of shear-thinning on the transient behaviour.

\section{1. 'Lift-up' effect and Reynolds stress mechanism}

It has been shown that, for in viscid shear flows, the streamwise velocity grows linearly with time for disturbances with no streamwise variation (Ellingsen \& Palm 1975). This growth mechanism has been labelled lift-up and vortex stretching, or more accurately vortex tilting. As explained by several authors (Butler \& Farrell 1992; Trefethen et al. 1993), for viscous shear flows the streamwise velocity grows linearly with time via the lift-up effect before being damped by the viscosity. In order to know growth mechanism in a non-Newtonian shear flow, it is helpful to recall the linearized momentum equation for the streamwise velocity component $u$ :

$$
\frac{\partial u}{\partial t}+w U^{\prime}=0
$$

in which $U$ is the steady mean flow and the prime $\left({ }^{\prime}\right)$ denotes $\mathrm{d} / \mathrm{d} z$. Because $w$ is independent of time, the streamwise velocity increases linearly with time. For disturbances independent of $x$, linearization of the in viscid vorticity equation gives

$$
\frac{\partial \omega}{\partial t}=U^{\prime} \frac{\partial w}{\partial y}
$$

where $\omega$ is the normal vorticity component. Note that $U^{\prime}$ is the vorticity of the mean flow. 
These two equations show that in the in viscid stage, the growth rates of $u$ and $\omega$ are proportional to $U^{\prime}$. For the Poiseuille flow of a shear-thinning fluid, with the decrease of $n$ the magnitude of $U^{\prime}$ decreases significantly in the region far away from the wall. Thus, it is natural that the lift-up effect becomes weaker with the increase of shear-thinning effect. In the work by Nouar et al. (2007), it is shown that the curves of $G(t)$ significantly decrease with the decrease of $n$. We infer that with the decrease of $n, G(t)$ decreases because the lift-up effect becomes less dominant in the region apart from the wall.

For the plane Couette flow of a shear-thinning fluid, the value $U^{\prime}$ is a constant for various $n$. Thus, the shear-thinning effect will not influence the energy growth in the in viscid stage. It only plays a role in the viscous stage. We can expect that with the increase of the shear-thinning effect, the transient energy growth will increase because of less viscosity. We anticipate that the influence of shear-thinning on the transient energy growth in the plane Couette flow may be different from that in the plane Poiseuille flow.

In addition to the lift-up effect, another growth mechanism, i.e. two-dimensional Reynolds stress mechanism, exists for shear flows (Pedlosky 1987). In an in viscid shear flow, the energy density equation is

$$
\frac{\partial \mathscr{E}}{\partial t}=-\frac{1}{2} \int_{-1}^{1} U^{\prime} \overline{u w} \mathrm{~d} z
$$

where $\mathscr{E}$ is the perturbation energy density and the overbar denotes an average of $x$. This expression indicates that a disturbance can extract energy from the mean shear. The growth rate of $\mathscr{E}$ is proportional to $U^{\prime}$. This mechanism has also been discussed by Orr associated with conservation in a strain field of spanwise perturbation vorticity (Orr 1907), and it will be referred to as the Orr mechanism. Farrel \& Ioannou (1993) studied the optimal excitation of three-dimensional perturbations in the viscous constant shear flow. The authors found that the optimal structures can be interpreted as combinations of two fundamental types of motion associated with the lift-up mechanism and the Orr mechanism.

For a power-law Couette flow, in the in viscid stage the index $n$ plays no role in the two-dimensional Reynolds stress mechanism. We also expect that the increase of shear-thinning effect increases the transient energy growth of the spanwiseindependent disturbances because of less viscosity after the in viscid stage.

\subsection{Pseudospectrum and response to external excitations}

We will study the transient behaviour of response to external excitations. Suppose a fluid system is driven by a harmonic signal of the form

$$
\boldsymbol{V}(x, y, z, t)=\mathrm{e}^{\mathrm{i}(-\omega t+\alpha x+\beta y)} \boldsymbol{v}(z) .
$$

It is easily verified that the response will be

$$
\boldsymbol{U}(x, y, z, t)=\mathrm{ie}^{\mathrm{i}(-\omega t+\alpha x+\beta y)}(\omega \mathscr{I}-\mathscr{L})^{-1} \boldsymbol{v}(z) .
$$

Here, $\mathscr{I}$ is the identity matrix. The solution operator $(\omega \mathscr{I}-\mathscr{L})^{-1}$ is known as the resolvent. The maximum value of amplification is equal to the norm of the resolvent:

$$
R(\alpha, \beta, \omega)=\max _{\boldsymbol{v} \neq 0} \frac{\|\boldsymbol{u}\|}{\|\boldsymbol{v}\|}=\left\|(\omega \mathscr{I}-\mathscr{L})^{-1}\right\| .
$$

Here, $\|\cdot\|$ denotes a norm on $\mathbb{C}^{N}$, max denotes the maximum. An eigenvalue of $\mathscr{L}$ is a number $\omega$ such that $\left\|(\omega \mathscr{I}-\mathscr{L})^{-1}\right\| \rightarrow \infty$. Generalizing this result leads naturally 
to the definition of ' $\epsilon-$ pseudospectrum' (Trefethen \& Embree 2005). For any $\epsilon \geqslant 0$, the ' $\epsilon$-pseudospectrum' of $\mathscr{L}$ is defined as

$$
\Lambda_{\epsilon}(\mathscr{L})=\left\{\omega \in \mathbb{C}:\left\|(\omega \mathscr{I}-\mathscr{L})^{-1}\right\| \geqslant \epsilon^{-1}\right\} .
$$

In mathematical meaning, the resolvent norm represents the amplification of response to external forcing. So, examining the structure of pseudospectra is helpful to understand the influence of shear-thinning effect on the non-modal stability of plane Couette flows. In the present paper, when investigating the response to spanwiseand streamwise-independent disturbances, we fix the wavenumber and the Reynolds number and plot the eigenvalues, the isolines of $\epsilon$-pseudospectra and the numerical range. The value of $\epsilon^{-1}$ corresponds to the maximal response $R(\omega, \alpha, \beta)$. The numerical range is defined as

$$
W(\boldsymbol{A})=\left\{\boldsymbol{x}^{*} \boldsymbol{A} \boldsymbol{x}: \boldsymbol{x} \in \mathbb{C}^{N},\|\boldsymbol{x}\|=1\right\},
$$

in which $\boldsymbol{A} \in \mathbb{C}^{N \times N}$. The numerical abscissa of $\boldsymbol{A}$ is defined as

$$
\omega(\boldsymbol{A})=\sup _{z \in W(\boldsymbol{A})} \operatorname{Re}(z)=\lim _{t \rightarrow 0} \frac{\mathrm{d}}{\mathrm{d} t}\left\|\mathrm{e}^{\boldsymbol{A} t}\right\| .
$$

The main application of the numerical range is to the analysis of energy growth for initial-value problems. The numerical abscissa of $-\mathrm{i} \mathscr{L}$ corresponds to the initial growth rate of the energy growth function (Reddy \& Henningson 1993; Trefethen \& Embree 2005). Reddy \& Henningson (1993) have presented the $\epsilon$-pseudospectra for the OS operator for a two-dimensional Newtonian Couette flow. The spectrum of OS operator displays a ' $\mathrm{Y}$ '-shaped structure, consisting of a left, a right and a middle part. The left and the right parts are symmetric about the imaginary axis, and each consists of two branches. In figure 6 , we plot the $\epsilon$-pseudospectra and the spectrum for various $n$ from 0.2 to 1.5 at $\alpha=1.0$ and $R e=1000$ for the disturbances which are uniform in the spanwise direction. We note that the spectrum in figure 6 includes the $\mathrm{OS}$ and $\mathrm{Sq}$ modes. The eigenvalues of the Sq operator locate in the imaginary axis and the middle lines of the left and the right branches.

Comparing the spectrum in figure 6 with different $n$, it shows that with the decrease of $n$ the spectrum of OS mode becomes more continuous. For example, in the left or right branch, the number of the OS eigenmodes is 6 for $n=1.5,8$ for $n=1.0$ and 10 for $n=0.5$. For $n=0.2$, the number of OS eigenmodes in the left or the right branch is greater than 14. Comparing the schematics in figure 6, it is observed that with the decrease of $n$, the boundary of $\epsilon=10^{-8}$ significantly extends in the $\omega$ plane. This result indicates that with the increase of shear-thinning, the intersection of the branches becomes more sensitive to perturbations. As $n$ decreases to 0.2, the region in the boundary of $\epsilon=10^{-8}$ covers both the intersection of branches and the middle branch. Additionally, the eigenvalues in the highly sensitive area become asymmetric about the imaginary axis. Comparing these figures, we found it interesting that the value of $n$ has almost no influence on the spectrum of the Sq modes. In each figure with different $n$, the left or the right branch always has eight Sq eigenvalues.

In figure 7, we plot the $\epsilon$-pseudospectra and the spectrum for the streamwiseindependent disturbances at $R e=1000$ for $n=1.0$ and 0.2. In the two figures, all the modes are located in the imaginary axis. Comparing figures $7(a)$ and $7(b)$, it is observed that the spectrum becomes more continuous with the decrease of $n$. The structures of the boundaries of $\epsilon$-pseudospectra are qualitatively similar for $n=1.0$ and 0.2 . In figures 6 and 7, the variation of $n$ has almost no influence on the numerical range and numerical abscissa. This result means that the shear-thinning/shear-thickening 

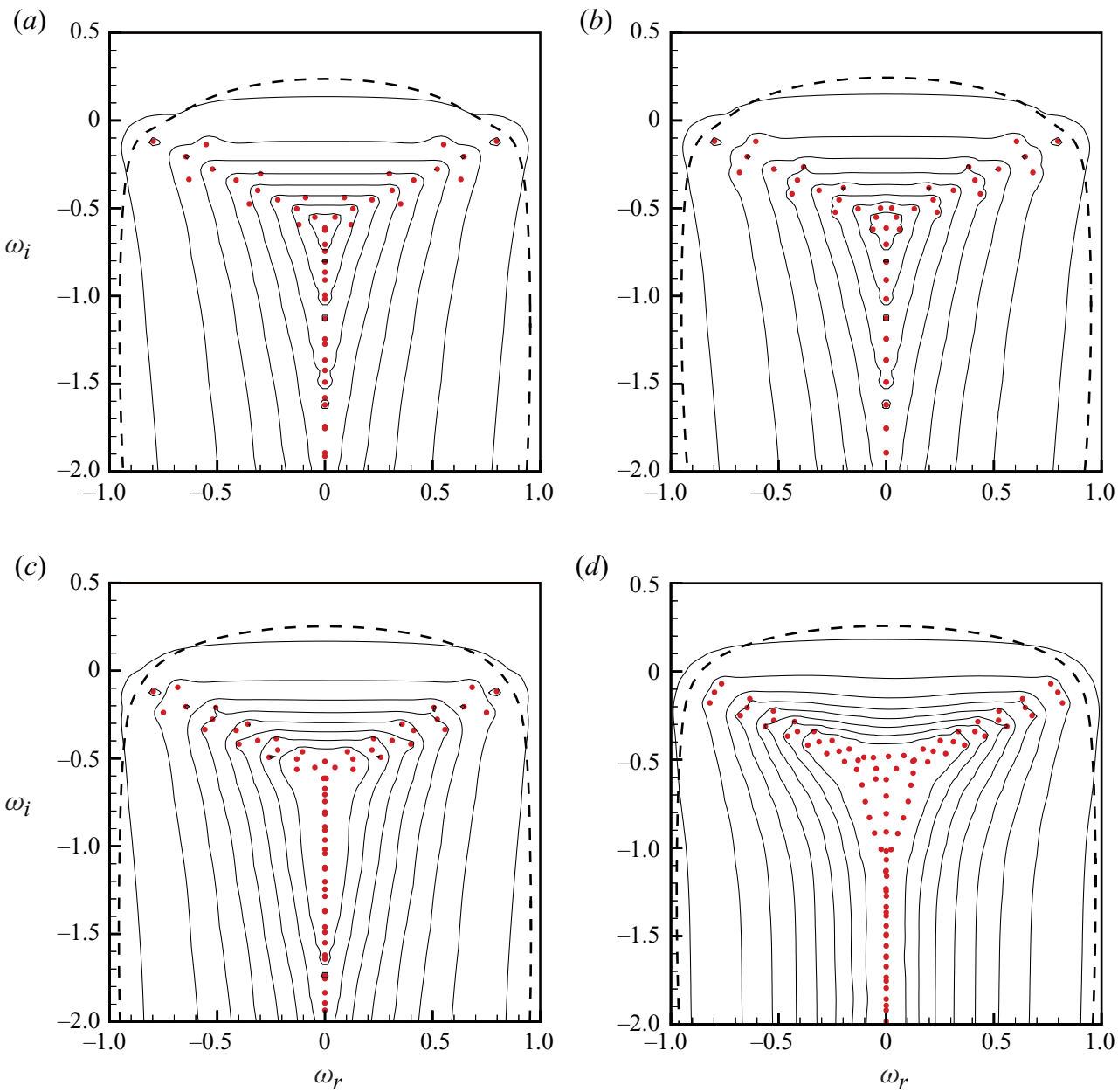

(d)

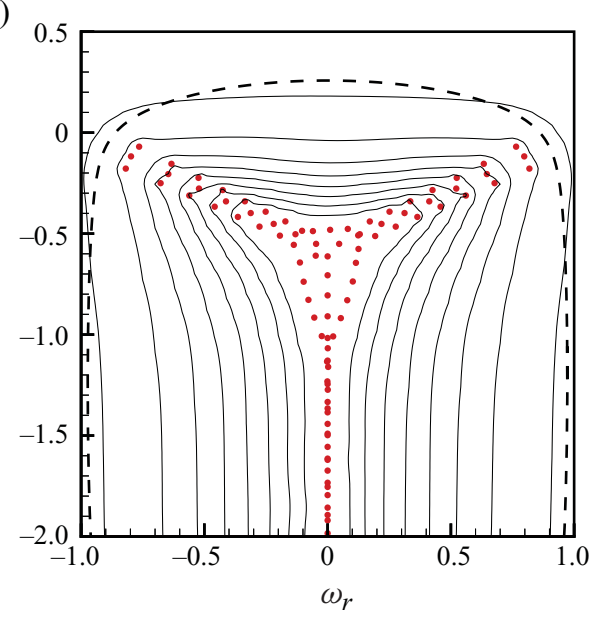

Figure 6. (Colour online) Pseudospectra for the plane Couette flow with $\alpha=1.0, \beta=0$ at $R e=1000$ for $(a) n=1.5$, (b) $n=1.0,(c) n=0.5,(d) n=0.2$. ๑: eigenvalues; --- : boundary of the numerical range; - : contours from outermost to innermost representing levels from $\epsilon=10^{-1}$ to $10^{-8}$.

effect only slightly influences the initial growth rate of the energy growth function for both the streamwise- and spanwise-independent disturbances.

In order to know the response to general excitations, we will examine the response to harmonic excitations with various wavelengths at different real frequencies. In figure 8 , we plot the level lines of amplifications to harmonic excitations of various frequencies in the $\alpha-\beta$ plane at $R e=300$ for $n=0.5$. At $\omega=0$, the maximal response is realized in the spanwise direction with the wavenumber of $\beta \approx 1$. As $\omega$ increases to 0.2 , in figure $8(b)$ the maximal response is shifted from the $\beta$-axis to $\beta \approx 2.0, \alpha \approx 0.5$. The figures show that with the increase of $\omega$, the maximal amplification decreases. As shown in these figures, as $\omega$ increases from 0 to 1.0 , the streamwise wavenumber of the most amplified disturbances increases from 0 to about 1.7, and the spanwise wavenumber increases from about 1.0 to 2.4. This result means that with the increase of $\omega$, the maximal response tends to occur in the shortwave range. 

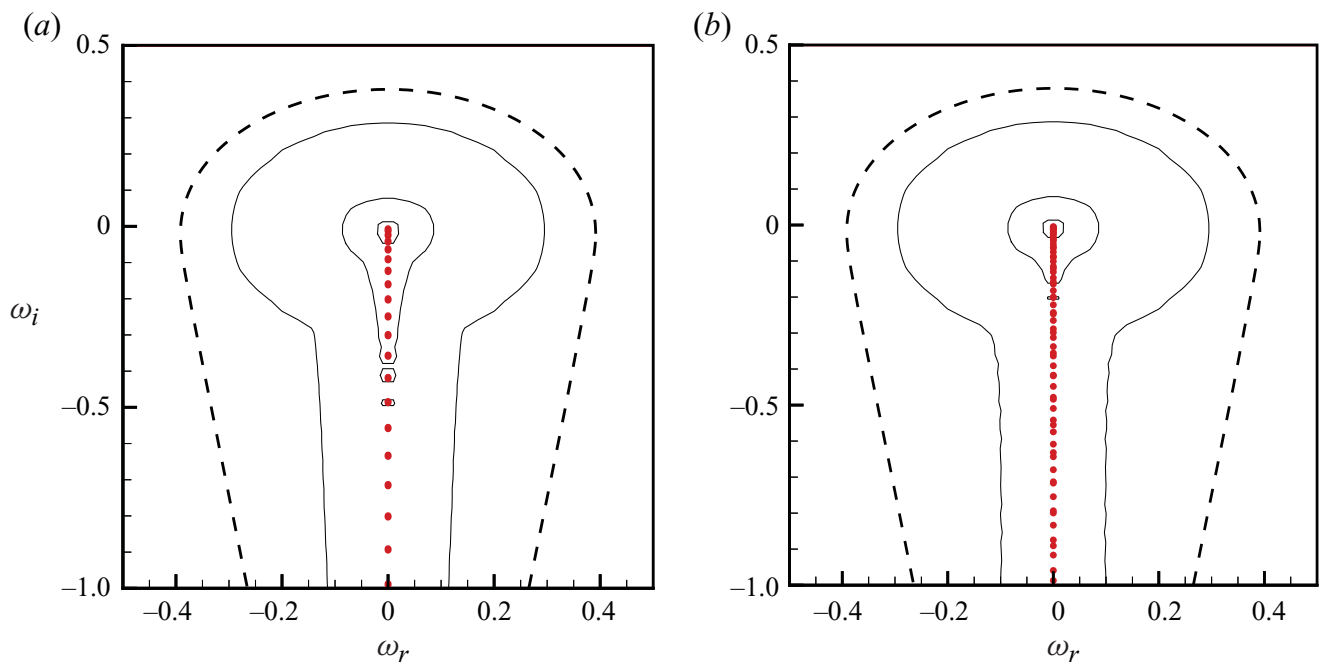

Figure 7. (Colour online) Pseudospectra for the plane Couette flow with $\alpha=0, \beta=2.0$ at $R e=1000$ for $(a) n=1,(b) n=0.2$. ๑: eigenvalues; --- : boundary of the numerical range; - : contours from outermost to innermost representing levels from $\epsilon=10^{-1}$ to $10^{-3}$.

We have presented the pseudospectra in the complex $\omega$ plane for several typical cases in figures 6 and 7. In the complex $\omega$ plane, we are interested in the resonance of the real axis, because it corresponds to external excitations at real frequencies. In order to obtain the maximal resonance to harmonic excitations, we need to examine the global behaviour of the operator $\mathscr{L}$ for all wavenumbers. We define the maximal amplifications of spanwise- and streamwise-independent disturbances as

$$
\begin{aligned}
& R_{\alpha}(\omega)=\max _{\forall \alpha \in \mathbb{R}, \beta=0} R(\alpha, \beta, \omega), \quad \omega \in \mathbb{R} \\
& R_{\beta}(\omega)=\max _{\alpha=0, \forall \beta \in \mathbb{R}} R(\alpha, \beta, \omega), \quad \omega \in \mathbb{R},
\end{aligned}
$$

in which $R(\alpha, \beta, \omega)$ has been defined in (5.6).

We have computed the maximal response at various Reynolds numbers and found that the structures of the response curves are similar. In figure $9(a)$, we plotted the curves of the maximal response to spanwise-independent external excitation versus the frequency with various $n$ at $R e=1000$. For each curve, the maximal amplification of the response is realized at $\omega=0$. With the increase of the magnitude of $\omega$, the amplitude of the response decreases. This result indicates that a time-independent forcing will result in the maximal response. With the decrease of $n$, the amplification significantly increases at all real frequencies. In figure $9(b)$, the curves of the streamwise wavenumbers of the maximal response versus the frequency are plotted. It is observed that for each curve the wavenumber increases with the increase of the magnitude of frequency. With the increase of shear-thinning, the wavelength of the most amplified spanwise-independent excitation increases at all frequencies. As explained in $\S 5.1$, the two-dimensional Reynolds stress mechanism is responsible for the transient growth of the spanwise-independent disturbances. In (2.12), the viscosity disturbance results in an anisotropic disturbance stress in the momentum equations in the $x$-and $z$-directions. The results in figure 9 indicate that in the presence of the viscosity disturbance, the shear-thinning makes the disturbance extract more energy from the mean shear. Thus, 
(a)

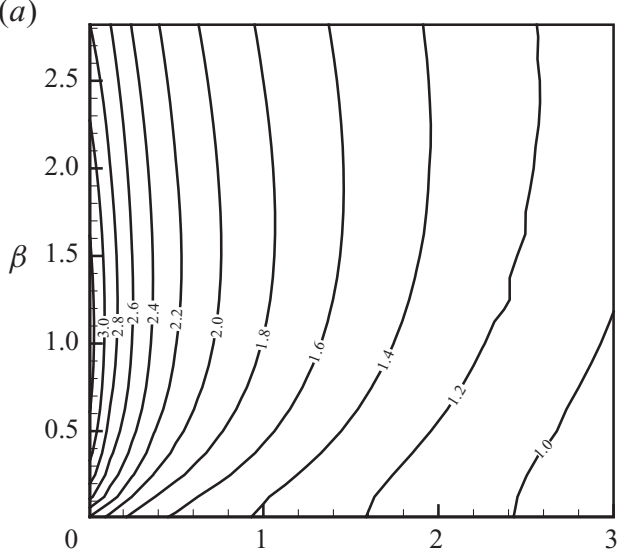

(c)

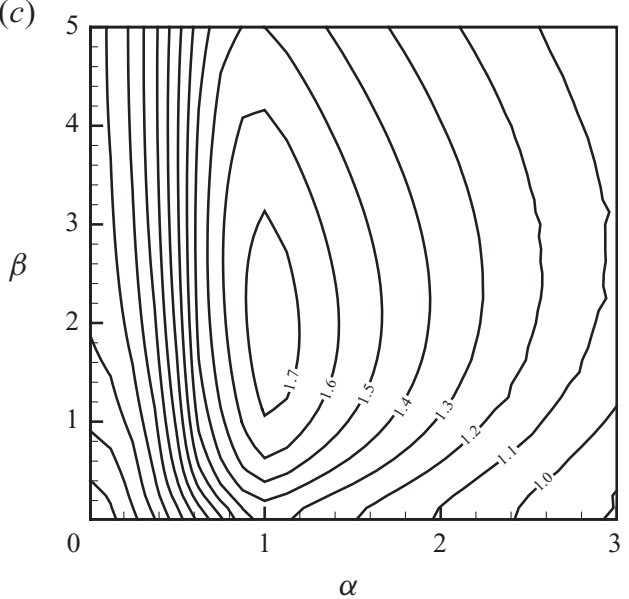

(b)

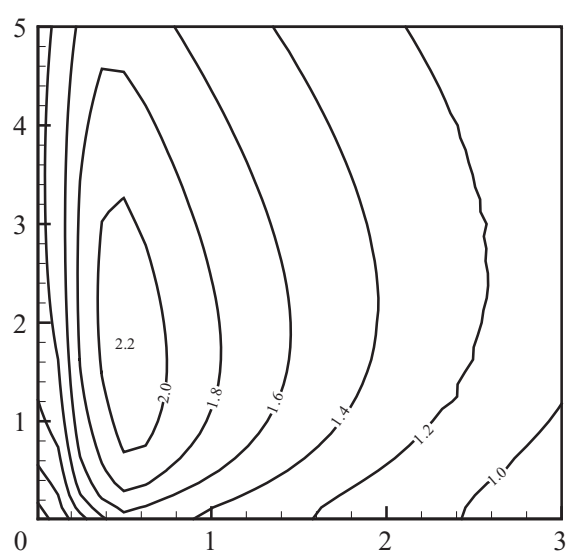

(d)

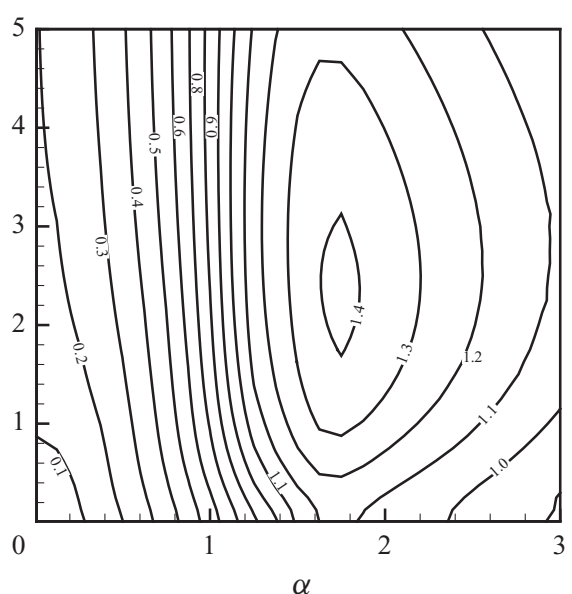

FIGURE 8 . The isolines of the logarithm of the response to three-dimensional disturbances in the $\alpha-\beta$ plane at $R e=300$ for $n=0.5$. (a) $\omega=0,(b) \omega=0.2,(c) \omega=0.5,(d) \omega=1.0$.
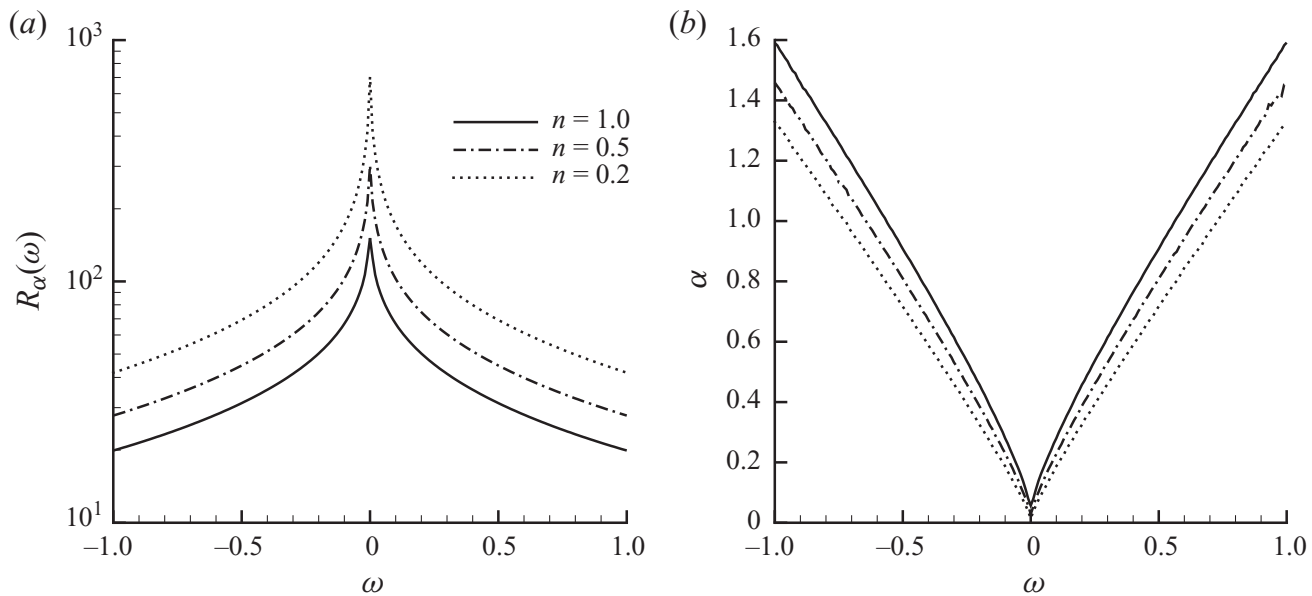

FIGURE 9. (a) The curves of the response to the continuous spanwise-independent external excitations versus the real frequency $\omega$ at $R e=1000$. (b) The curves of the streamwise wavenumber corresponding to the maximal response at $R e=1000$. 

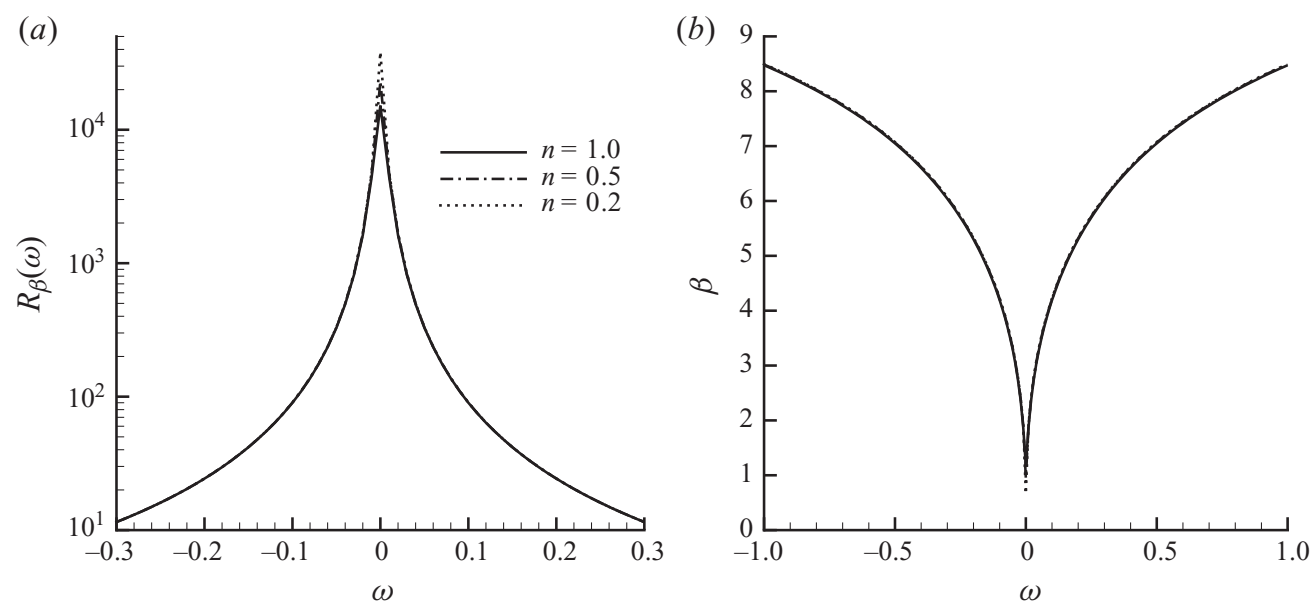

FigurE 10. (a) The curves of the response to the continuous streamwise-independent external excitations versus the real frequency $\omega$ at $R e=1000$. (b) The curves of the spanwise wavenumber corresponding to the maximal response at $R e=1000$.

the response to external spanwise-independent excitations significantly increases with the increase of shear-thinning.

In figure 10(a), the curves of the maximal response to streamwise-independent external excitation versus the frequency are presented for various $n$ at $R e=1000$. In this figure, like the spanwise-independent case, the most amplified response to streamwise- independent excitation is realized at $\omega=0$. Additionally, the amplification significantly decreases with the increase of the magnitude of $\omega$. Comparing the curves with the same value of $n$ in figures $10(a)$ and $9(a)$, it is shown that the streamwiseindependent excitation is amplified more than the spanwise-independent one. Figure 10(a) shows that shear-thinning only increases the amplification in a very narrow range of low frequencies. In figure $10(b)$, the curves of the wavenumber of the most amplified response are almost indistinguishable for various $n$, except that the frequency is very low. These results show that shear-thinning has almost no effect on the response to streamwise-independent disturbances in the range of medium or high frequency.

For a Couette flow, the optimal input of harmonic disturbances is uniform in the streamwise direction at $\omega=0$. The spatial structures of the optimal input of harmonic excitation and the maximum response are shown in figure 11 for $n=0.5$ at $R e=1000$. As here the optimal frequency is $\omega=0$, only plots of input and output are needed. We computed the velocity fields of the optimal input and output. The results show that the streamwise components of input disturbances are very weak, and the normal and spanwise velocity components have most of the perturbation kinetic energy. For the optimal output, the magnitudes of normal and spanwise velocity components are slight in comparison with the streamwise component. The velocity field $v e_{y}+w e_{z}$ associated with the optimal input is plotted in figure 11(a) for $n=0.5$ at $R e=1000$. The flow field is characterized by two counter-vortices with the centre near $z=0$. The optimal output of the amplitude of streamwise velocity component $u$ is plotted in figure $11(b)$. At the optimal time, the amplitude of the optimal response is 23127 . At this time, the two counter-vortices transform into streaks. With the increase of shear-thinning, the amplitude of response increases and the structures of the optimal input and output disturbances are similar to the Newtonian case. The flow fields in 

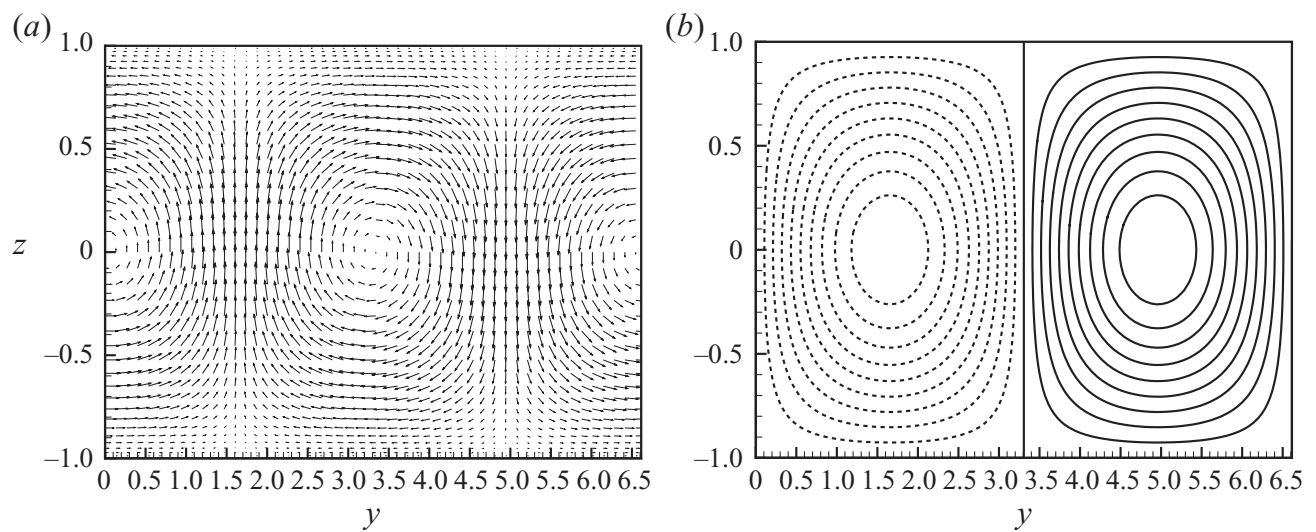

FIGURE 11. Cross-stream $(y-z)$ view of the optimal input and output fields of the response to the harmonic forcing problem at $R e=1000, \beta=0.95$ and $n=0.5$. (a) the velocity components of $v$ and $w$ of the input. (b) isoline of $u$ of the output.

figures 11(a) and 11(b) imply that the dominant physical mechanism for the response to excitation is the lift-up effect. With the increase of shear-thinning, the streamwiseindependent disturbance has a higher amplitude of response because of less viscosity. Comparing the flow fields with various $n$, it is found that with the increase of shearthinning, the optimal wavenumber becomes smaller. For example, as $n$ decreases from 0.5 to 0.2 , the optimal wavenumber decreases from $\beta=0.95$ to 0.66 .

\subsection{Transient growth and optimal disturbances}

In this subsection, we will focus on the effect of non-Newtonian viscosity on the response of initial conditions. The greatest amplification of the initial condition at time $t$ is given by the growth function as

$$
G(t)=\max _{\boldsymbol{u}(0) \neq 0} \frac{\|\boldsymbol{u}(t)\|^{2}}{\|\boldsymbol{u}(0)\|^{2}}=\left\|\mathrm{e}^{-\mathrm{i} \mathscr{L} t}\right\|^{2} .
$$

We can define the growth maximized over time as

$$
G_{\max }=G\left(t_{\max }\right)=\max G(t), \quad t \geqslant 0 .
$$

Reddy \& Henningson (1993) considered three types of behaviours for the plane Poiseuille flow of Newtonian fluids. If the Reynolds number is less than the critical energy Reynolds number $R e_{g}$, then $G(t) \leqslant 1$ for all time. In this case, $G_{\max }=1$ and $t_{\max }=0$. If the Reynolds number exceeds the critical Reynolds number $R e_{c}$ such that the operator $\mathscr{L}$ has an unstable eigenvalue, then $G_{\max } \rightarrow \infty$ at $t_{\max } \rightarrow \infty$. If $R e_{g}<R e<R e_{c}$, the flow is linearly stable but has a transient energy growth. For general stability problems, these three types of behaviours should be considered. However, the plane Couette flow is linearly stable in general. When examining the characteristics of the transient growth, we only need to consider the case of $R e>R e_{g}$.

In this subsection, we first examine the energy growth of spanwise- and streamwiseindependent disturbances. Figures $12(a)$ and 12(b) plot the curves of the growth function $G(t)$ for spanwise-independent disturbances with various $n$ at $R e=300$ and 1000 , respectively. As we discussed in $\S 5.1$, the two-dimensional Reynolds stress mechanism is responsible for the rapid transient growth of the spanwise-independent disturbances. In figure 12, each curve shows that the disturbance experiences a transient growth and then decays after $t_{\max }$. With the decrease of $n$, the plane Couette 

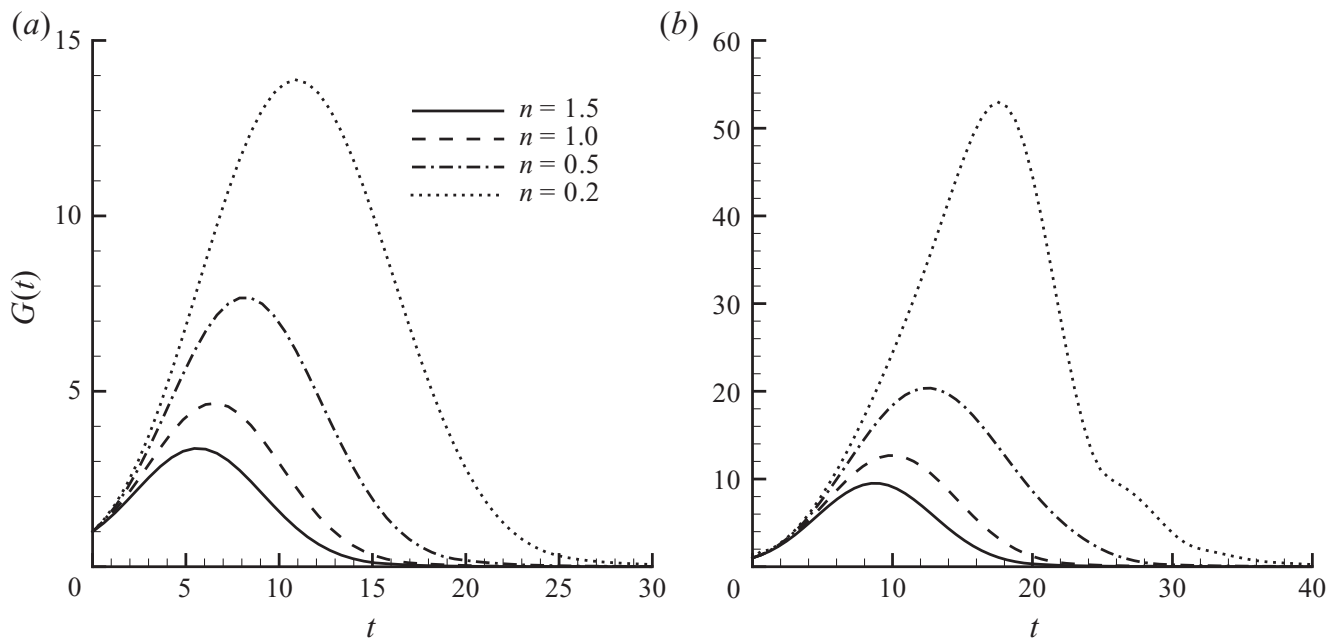

FiguRE 12. Transient energy growth function $G(t)$ versus time for spanwise-independent disturbances with various $n$ at $\alpha=1.0$. (a) $R e=300$, (b) $R e=1000$.
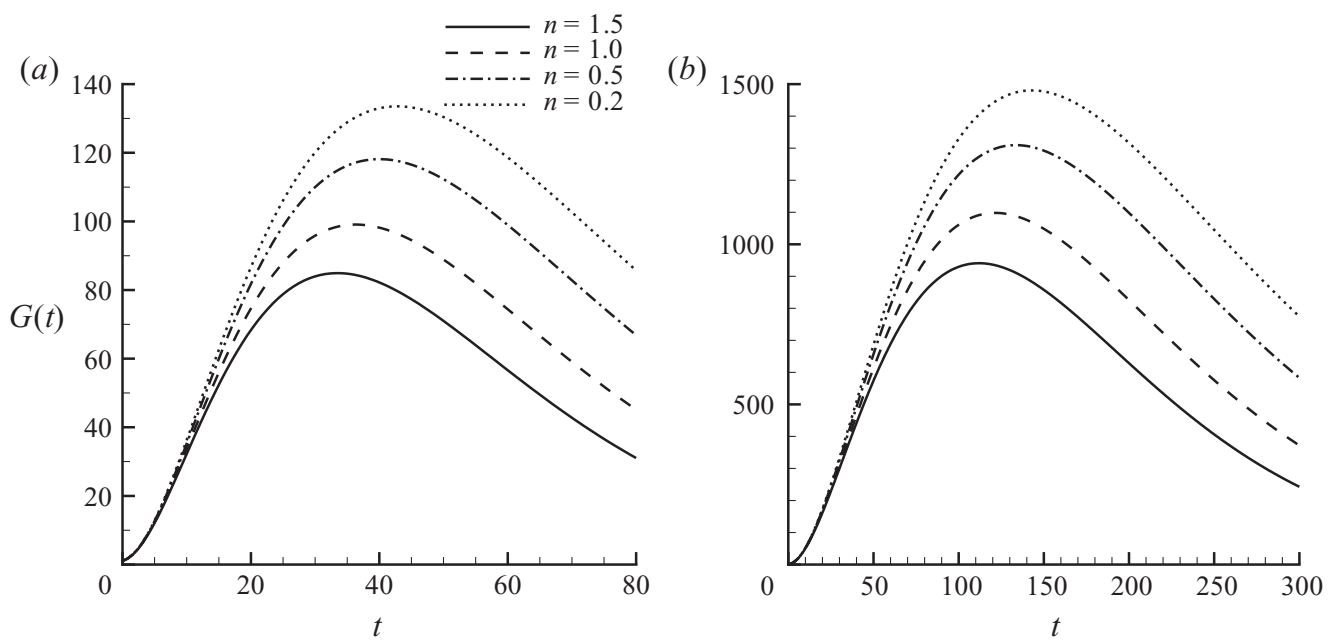

FiguRE 13. Transient energy growth function $G(t)$ versus time for streamwise-independent disturbances with various $n$ at $\beta=2.0$. (a) $R e=300$, (b) $R e=1000$.

flow has a greater transient energy growth. This result is consistent with the discussion in $\S 5.1$ that shear-thinning is destabilizing because of less viscosity. In figure 12, it is observed that for more shear-thinning fluids, the spanwise-independent disturbances need more time to reach the maximal energy growth. For the present problem, we are interested in the transient behaviours at initial stage. In figure 12(a), for a low Reynolds number of $R e=300$, with the decrease of $n$, the temporal growth rate of $G(t)$, i.e. $\mathrm{d} G(t) / \mathrm{d} t$, slightly increases at $t=0$. However, in figure $12(b)$ for $R e=1000$, the curves with various $n$ are almost undistinguishable at the initial stage. This result is consistent with the discussion in $\S 5.1$ that shear-thinning does not influence the two-dimensional Reynolds stress mechanism at the initial stage.

Figures $13(a)$ and $13(b)$ plot the curves of the growth function $G(t)$ for streamwiseindependent disturbances with various $n$ at $R e=300$ and 1000 , respectively. In figure 13, each curve shows that the disturbance experiences a transient growth and 

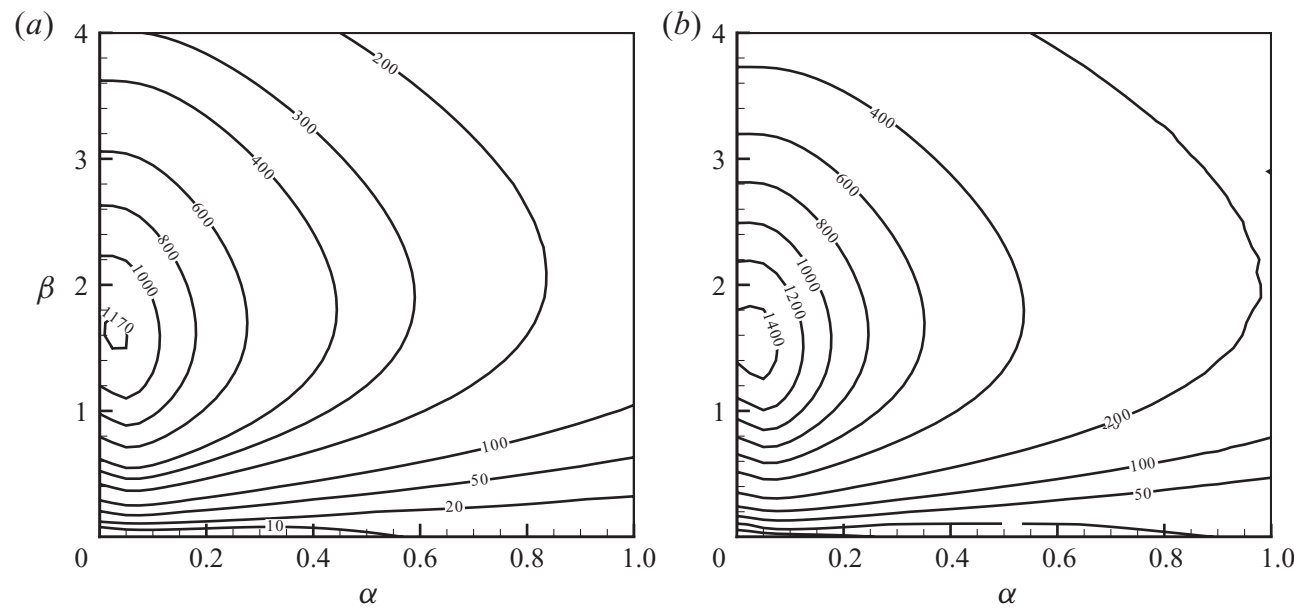

FIGURE 14. Level lines of the maximum energy growth $G_{\max }$ in the $\alpha-\beta$ plane at $R e=1000$. (a) Newtonian case of $n=1.0,(b)$ shear-thinning case of $n=0.5$.

then decays after $t_{\max }$. Moreover, with the decrease of $n$ the streamwise-independent disturbance has a greater maximal energy growth $G_{\max }$ and needs more time to reach the maximal growth. Comparing the curves with the same index $n$ in figures $13(a)$ and $13(b)$, it is shown that the energy growth $G(t)$ is much greater at $R e=1000$ than that at $R e=300$. In these two figures, the curves with various $n$ are almost undistinguishable at the initial stage. This result is consistent with the discussion in $\S 5.1$ that the non-Newtonian viscosity does not affect the lift-up mechanism for the Couette flow in the in viscid stage. Comparing figure 12 with figure13, we found that the transient energy growth of the streamwise-independent disturbances is much higher than the spanwise-independent one.

Chikkadi et al. (2005) have studied the characteristics of the transient energy growth for the plane Poiseuille flow of a Carreau fluid. The authors only considered the stratification of viscosity and neglected the perturbation of viscosity. Their results indicate that the shear-thinning effect has little influence on the transient growth of spanwise disturbances. Nouar et al. (2007) have revisited the same problem as Chikkadi et al. (2005). The authors have accounted for a non-vanishing viscosity disturbance, which yields an anisotropic disturbance stress tensor. The results of Nouar et al. (2007) show that shear-thinning effect significantly reduces the maximal energy growth of streamwise-independent disturbances.

For the present problem, we have taken into account the perturbation of viscosity. For the Couette flow, there is no stratification of viscosity; so the effect of nonNewtonian viscosity only includes an anisotropic disturbance stress tensor. As shown in figures 12 and 13, the shear-thinning effect significantly increases the transient energy growth of the disturbances. It seems that the shear-thinning effect on the plane Couette flow is opposite to that on the plane Poiseuille flow reported by Nouar et al. (2007). This result is consistent with the prediction in $\S 5.1$ that the shear-thinning is destabilizing for the plane Couette flow.

We have examined the transient behaviours of the streamwise- and spanwiseindependent disturbances. In order to know the energy growth of oblique disturbances, we will present the maximal energy growth $G_{\max }$ in the $\alpha-\beta$ plane. In figure 14 , isolines of $G_{\max }$ are plotted for $n=1.0$ and 0.5 at $R e=1000$. The structures of the level lines 
(a)

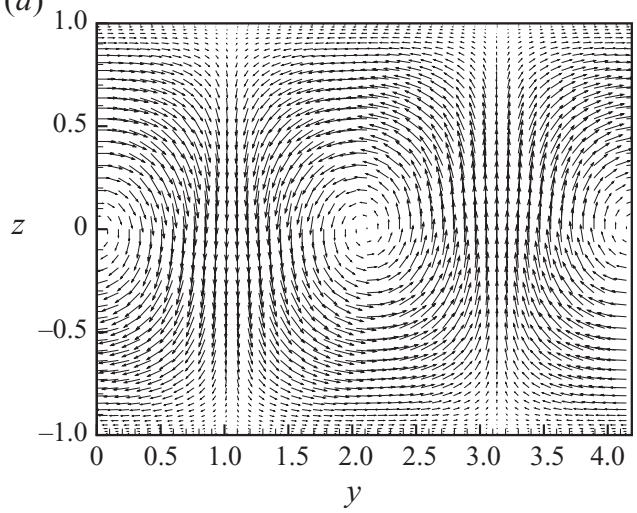

(b)

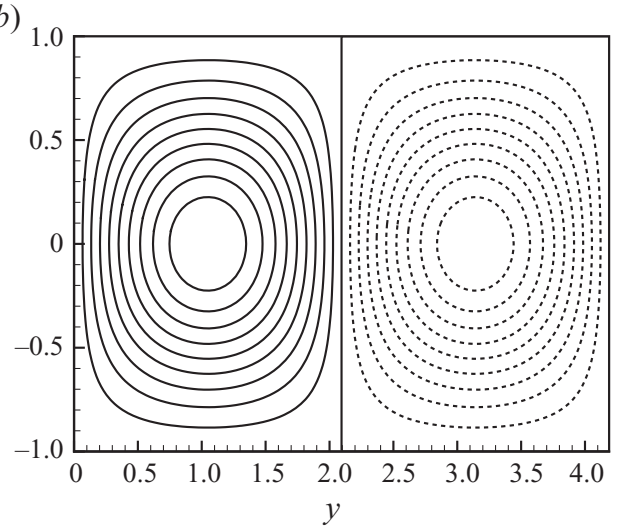

FIGURE 15. Cross-stream $(y-z)$ view of the optimal input and output fields of the initial problem at $R e=1000, \beta=1.5$ and $n=0.5$. (a) the vectors of $v$ and $w$ components at $t=0$. (b) isolines of $u$ component at optimal time $t_{\max }=183.6, G_{\max }=1685.1$.

of $G_{\max }$ are similar in these two cases. In the $\alpha-\beta$ plane, we can define the optimal energy growth as

$$
G_{\text {opt }}=\max _{\alpha, \beta} G_{\max }(\alpha, \beta) \quad \text { at } \quad t=t_{\text {opt }} .
$$

For the plane Poiseuille flow, the optimal energy growth is realized in the spanwise direction $(\alpha=0)$ (Reddy \& Henningson 1993). However, for the plane Couette flow, the most amplified disturbance does not located in the $\beta$-axis. In figures 14(a) and $14(b)$, the optimal amplification is in the form of non-streamwise uniform disturbances, but having a very large streamwise wavelength. As shown in figure 14(a), the optimal energy growth of the plane Couette flow is realized at the point of $\alpha \approx 0.025$ and $\beta \approx 1.6$ for $n=1.0$. For $n=0.5$, the optimal disturbance shifts to the point of $\alpha \approx 0.04$ and $\beta \approx 1.5$.

In order to know more about the influence of shear-thinning on the energy growth of optimal disturbances, we plot the flow fields at the initial time $t=0$ and the optimal time $t_{\max }$ for two typical cases, i.e. the streamwise- and spanwise-independent disturbances. In figure 15, we plot the flow field for the optimal streamwiseindependent disturbance at $\operatorname{Re}=1000, \beta=1.5$ for $n=0.5$. At the initial time $t=0$ and optimal time $t_{\max }$, the flow fields are characterized by two counter-rotating vortices and the optimal streaks. These flow structures of the shear-thinning case are qualitatively similar to that of the Newtonian case. As explained in many previous works, the streamwise velocity grows linearly with time via the lift-up mechanism before being damped by the viscosity. The structures of the flow at the $t=0$ and $t_{\max }$ are also very similar to the optimal input and output of harmonic excitations in figure 11. The difference is that the spanwise wavelength of the initial value problem in figure 15 is smaller than that in figure 11 . We have also computed the flow fields of streamwise-independent disturbances for various values of $n$; it is found that the structures of the flow fields and the wavelength are only slightly changed with $n$. It seems that the non-Newtonian viscosity does not influence the streaky structure of the streamwise-independent disturbances.

In figure 16, we plot the isolines of the streamfunction of the spanwise-independent disturbances at initial time and optimal time for $n=1.0$ and 0.5. As shown in figures $16(a)$ and 16(c), the streamlines of the initial flow field tilt opposite to 

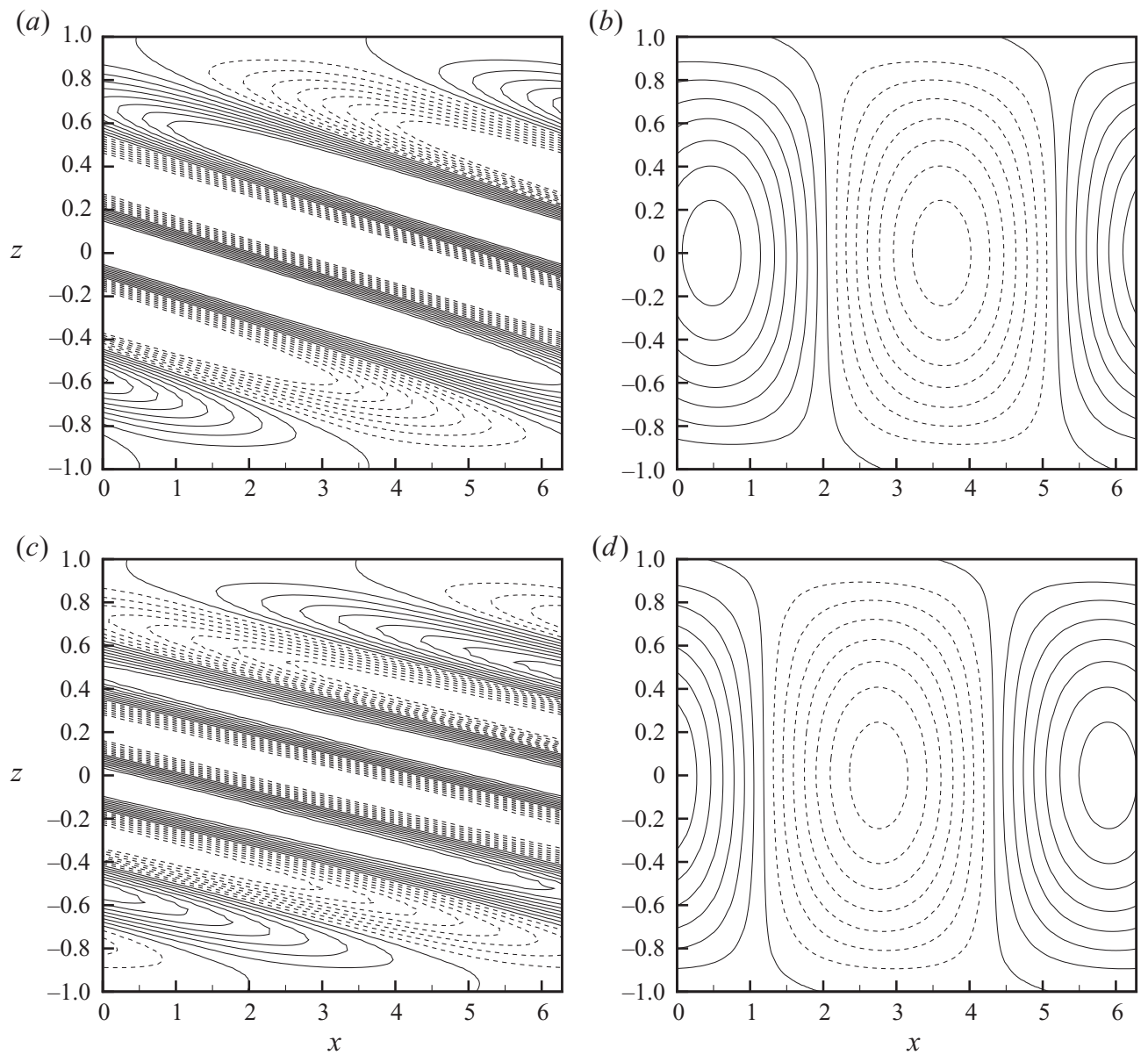

FIGURE 16. Flow patterns of the streamfunction for the spanwise-independent disturbances at $R e=1000$ for $n=1.0$ and 0.5. (a) Flow filed at initial time, $(b)$ flow field at optimal time for $n=1.0, G_{\max }=13.0, t_{\max }=8.7, \alpha=1.23 ;(c)$ flow filed at initial time, $(d)$ flow field at optimal time for $n=0.5, G_{\max }=20.5, t_{\max }=11.7, \alpha=1.10$.

the mean flow. As explained by the two-dimensional Reynolds stress mechanism, the disturbance gains energy from the mean shear. Comparing figure 16(a) with figure 16(c), it is observed that with the increase of shear-thinning, the streamlines tilt more opposite to the direction of the mean flow. This means that for a more shear-thinning fluid, the disturbance can extract more energy from the mean flow. At the optimal time, the structures of the flow fields are similar for $n=1.0$ and $n=0.5$. As shown in figures $16(b)$ and $16(d)$, the flow patterns become upright. In this case, the disturbance gains no energy from the mean shear. After the optimal time, we found that the flow patterns of the streamlines tilt to the streamwise direction. At this stage, the disturbance energy is returned to the mean flow.

\section{Conclusions}

In this paper, linear stability in the plane Couette flow of a power-law fluid has been investigated using the eigenvalue analysis, the energy method and the non-modal stability theory. We focus on the effect of shear-thinning on the stability of the flow. 
For the plane Couette flow of a power-law fluid, there is no stratification of viscosity. The effect of non-Newtonian viscosity is in the form of an anisotropic of stress tensor of disturbance.

The eigenvalue analysis is used to investigate the long time behaviour of the disturbances. We have studied the temporal growth rate of the least stable mode for both the spanwise- and streamwise-independent disturbances. It is found that the plane Couette flow is always stable for shear-thinning fluids at rather high Reynolds numbers. This suggests that, like the Newtonian case, the plane Couette flow of a shear-thinning fluid is always stable at all Reynolds number. For both the spanwise- and streamwise-independent disturbances, the temporal growth rate decreases with the increase of the wavenumber. For spanwise-independent disturbances, the shear-thickening effect only slightly stabilizes the system. However, the shear-thinning effect significantly destabilizes the system in the medium and shortwave ranges. For streamwise-independent disturbances, the shear-thinning effect significantly destabilizes the plane Couette flow.

We have studied the condition for no energy growth using the energy method. The critical energy Reynolds number $R e_{g}$ below which there is no energy growth was determined by searching the maximal eigenvalue of $\left(\mathscr{L}-\mathscr{L}^{*}\right) / 2$. The streamwise-independent disturbances are more unstable than the spanwiseindependent disturbances for all the index $n$. With the increase of $n$, the plane Couette flow becomes more stable for both the streamwise- and spanwise-independent disturbances.

The main focus of the present paper is the non-modal stability of the plane Couette flow. We study the response to external excitations and initial conditions. The response to external excitations is characterized using the $\epsilon$-pseudospectrum, and the response to initial conditions is described by the energy growth function $G(t)$. For both the streamwise- and spanwise-independent disturbances, the maximal response to external excitations is realized at $\omega=0$. The shear-thinning effect significantly increases the amplification of response for the spanwise-independent disturbances at both low and high frequencies. However, the response to streamwise-independent excitations is insensitive to shear-thinning at high frequency. When fixing $\omega$, we present the maximal response to external excitation in the $\alpha-\beta$ plane. For $\omega=0$, the most amplified disturbance is streamwise uniform. For $\omega>0$, the most amplified disturbance becomes oblique. The increase of $n$ reduces the response of oblique disturbances in the $\alpha-\beta$ plane.

We have studied the effect of shear-thinning on the transient energy growth of initial conditions. The results show that when the Reynolds number exceeds $R e_{g}$, the disturbances experience a transient growth and then an exponential decay. For the plane Poiseuille flow, the optimal disturbance is streamwise uniform. However, for the plane Couette flow of a Newtonian or non-Newtonian fluid, the optimal response is achieved by a slightly oblique disturbance with a very large streamwise wavelength. Both the lift-up effect and the two-dimensional Reynolds stress mechanism can be responsible for the energy growth of the initial disturbances. With the increase of shear-thinning, the transient energy growth increases for both the streamwise- and spanwise-independent disturbances.

All results in the present paper show that in the absence of a stratification of viscosity, the effect of shear-thinning is destabilizing for the plane Couette flow. This result is different to that for the plane Poiseuille flow, in which a stratification of viscosity plays an important role and the shear-thinning effect appears to be stabilizing. In order to explain the difference between the present problem and the 
plane Poiseuille flow, we examine the energy budget of the Orr-Sommerfeld equation for the eigenvalue problem. For a general plane shear flow, shear-thinning has a dual effect. The viscosity stratification arising from shear-thinning is stabilizing, and the effect of the viscosity disturbance is destabilizing. For the plane Poiseuille flow, the stabilizing effect is dominant. For the plane Couette flow, there is no viscosity stratification. So, the total effect of shear-thinning is destabilizing. We also examine the effect of shear-thinning on the lift-up mechanism and the two-dimensional Reynolds stress mechanism which are responsible for the transient energy growth. For the plane Poiseuille flow, with the decrease of $n$ the magnitude of $U^{\prime}$ decreases in the region far away from the walls. So, the lift-up mechanism and the two-dimensional Reynolds stress mechanism become weaker with the increase of shear-thinning. However, for the Couette flow, $U^{\prime}$ is independent of the index $n$. With the increase of shear-thinning, the transient energy growth arising from these two mechanisms increases because of less viscosity.

The authors thank the National foundation of China for financial support for this research $(50890182,11072249$ and 10772185). The authors also gratefully acknowledge the referees for their many helpful comments.

\section{REFERENCES}

Bergström, L. B. 2003 Transient growth of small disturbances in a Jeffrey fluid flowing through a pipe. Fluid Dyn. Res. 32, 29-44.

Bird, R. B., Armstrong, R. C. \& Hassager, O. 1977 Dynamics of Polymeric Liquids: Fluid Mechanics, vol. 1. Wiley.

Bird, R. B., Stewart, W. E. \& Lightfoot, E. N. 2002 Transport Phenomena. Wiley.

Butler, K. M. \& Farrell, B. F. 1992 Three-dimensional optimal perturbations in viscous shear flow. Phys. Fluids A 4, 1637-1650.

Canuto, C., Hussaini, M. Y., Quarteroni, A. \& Zang, T. A. 1993 Spectral Method in Fluid Dynamics. Springer.

Chikkadi, V., Sameen, A. \& Govindarajan, R. 2005 Preventing transition to turbulence: a viscosity stratification does not always help. Phy. Rev. Lett. 95, 264504-1-264504-4.

Davey, A. \& Drazin, P. G. 1969 The stability of Poiseuille flow in a pipe. J. Fluid Mech. 36, 209-218.

Davey, A. \& Nguyen, H. P. F. 1971 Finite-amplitude stability of pipe flow. J. Fluid Mech. 45, 701-720.

Eldabe, N. T. M., EI-Sabbagh, M. F. \& EI-SaYed, M. A.-S. 2007 The stability of plane Couette flow of a power-law fluid with viscous heating. Phys. Fluids 19, 094107-1-094107-9.

Ellingsen, T. \& Palm, E. 1975 Stability of linear flow. Phys. Fluids 18, 487-488.

FARrel, B. F. \& IOANNOU, P. J. 1993 Optimal excitation of three-dimensional perturbations in viscous constant shear flow. Phys. Fluids A 6,1390-1400.

Hoda, N., Jovanović, M. R. \& KumaR, S. 2008 Energy amplification in channel flows of viscoelastic fluids. J. Fluid Mech. 601, 407-424.

Hoda, N., Jovanović, M. R. \& Kumar, S. 2009 Frequency response of streamwise-constant perturbations in channel flows of Oldroyd-B fluids. J. Fluid Mech. 625, 411-434.

Hwang, Y. \& Cossu, C. 2010 Amplification of coherent streaks in the turbulent Couette flow: an input-output analysis at low Reynolds number. J. Fluid Mech. 643, 333-348.

Jovanović, M. R. 2004 Modeling, analysis, and control of spatially distributed systems. PhD thesis, University of California, Santa Barbara, CA.

Jovanović, M. R. \& BAMIEH, B. 2005 Componentwise energy amplification in channel flows. J. Fluid Mech. 534, 145-183.

Kiton, O., Nakabyashi, K. \& Nishimura, F. 2005 Experimental study on mean velocity and turbulence characteristics of plane Couette flow: low-Reynolds-number effects and large longitudinal vortical structure. J. Fluid Mech. 539, 199-227. 
Kline, S. J., Reynolds, W. C., Schraub, F. A. \& Runstadler, P. W. 1967 The structure of turbulent boundary layers. J. Fluid Mech. 30, 741-773.

LANDAHL, M. T. 1975 Wave breakdown and turbulence. SIAM J. Appl. Maths 28, 735-756.

LANDAHL, M. T. 1980 A note on an algebraic instability of inviscid parallel shear flows. J. Fluid Mech. 98, 243-251.

Malerud, S., MÅløy, K. J. \& Goldburg, W. I. 1995 Measurements of turbulent velocity fluctuations in a planar Couette cell. Phys. Fluids 7, 1949-1955.

Malik, S. V. \& Hooper, A. P. 2005 Linear stability and energy growth of viscosity stratified flows. Phys. Fluids 17, 024101-1-024101-8.

Matsubara, M. \& Alfredsson, P. H. 2001 Disturbance growth in boundary layers subjected to free-stream turbulence. J. Fluid Mech. 430, 149-168.

Nouar, C., Bottaro, A. \& Brancher, J. P. 2007 Delaying transition to turbulence in channel flow: revisiting the stability of shear-thinning fluids. J. Fluid Mech. 592, 177-194.

Nouar, C. \& FrigaARD, I. 2009 Stability of plane Couette-Poiseuille flow of shear-thinning fluid. Phys. Fluids 21, 064104-1-064104-13.

Nouar, C., Kabouya, N., Dusek, J. \& Mamou, M. 2007 Modal and non-modal linear stability of the plane Bingham-Poiseuille flow. J. Fluid Mech. 577, 211-239.

OrR, W. M. F. 1907 The stability or instability of the steady motions of a perfect liquid and of a viscous liquid. Proc. R. Irish Acad. A 27, 9-138.

OrszaG, S. A. 1971 Accurate solution of the Orr-Sommerfeld stability equation. J. Fluid Mech. 50, 689-703.

Pedlosky, J. 1987 Geophysical Fluid Dynamics, 2nd edn. Springer.

Pinarbasi, A. \& Imal, M. 2005 Viscous heating effects on the linear stability of Poiseuille flow of an inelastic fluid. J. Non-Newtonian Fluid Mech. 127, 67-71.

Ranganathan, B. T. \& Govindarajan, R. 2001 Stabilization and destabilization of channel flow by location of viscosity-stratified fluid layer. Phys. Fluids 13, 1-3.

Reddy, S. \& Henningson, D. S. 1993 Energy growth in viscous channel flows. J. Fluid Mech. 252, 209-238.

Reddy, S., Schmid, P. J., Baggett, J. S. \& Henningson, D. S. 1998 On stability of streamwise streaks and transition thresholds in plane channel flows. J. Fluid Mech. 365, 269-303.

Roberts, S. A. \& Kumar, S. 2006 Stability of creeping Couette flow of a power-law fluid past a deformable solid. J. Non-Newtonian Fluid Mech. 139, 93-102.

Romanov, V. A. 1973 Stability of plane-parallel Couette flow. Funct. Anal. Appl. 7, 137-146.

Schmid, P. J. \& Henningson, D. S. 1994 Optimal energy growth in Hagen-Poiseuille flow. J. Fluid Mech. 277, 197-225.

Schmid, P. J. \& Henningson, D. S. 2001 Stability and Transition in Shear Flows. Springer.

Schmid, P. J. 2007 Non-modal stability theory. Annu. Rev. Fluid Mech. 39, 129-162.

Thomas, L. H. 1953 The stability of plane Poiseuille flow. Phys. Rev. 91, 780-783.

Tillmark, N. \& Alfredsson, P. H. 1992 Experiments on transition in plane Couette flow. J. Fluid Mech. 235, 89-102.

Trefethen, L. N. \& Embree, M. 2005 Spectra and Pseudospectra: the Behavior of Nonnormal Matrices and Operators. Princeton University Press.

Trefethen, L. N., Trefethen, A. E., Reddy, S. C. \& Driscoll, T. A. 1993 Hydrodynamics stability without eigenvalues. Science 261, 578-584.

Wygnanski, I. \& Champagne, F 1973 On transition in a pipe. Part 1. The origin of puffs and slugs and the flow in a turbulent slug. J. Fluid Mech. 59, 281-335.

Wygnanski, I., Sokolov, M. \& Friedman, D. 1975 On transition in a pipe. Part 2. The equilibrium puff. J. Fluid Mech. 69, 283-304. 